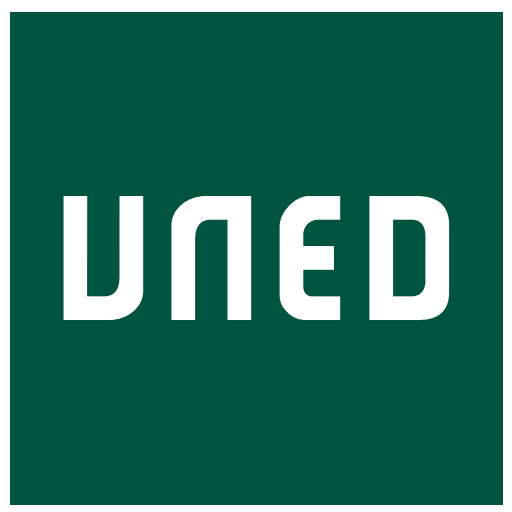

\title{
SIAMES
}

\section{Social Impact Advisor and Measurement System}

\author{
Autor
}

Daniel Hernández Marín

\section{Directoras}

Dr. ${ }^{a}$ Ángeles Manjarrés Riesco

Dr. ${ }^{a}$ Marta Solórzano García

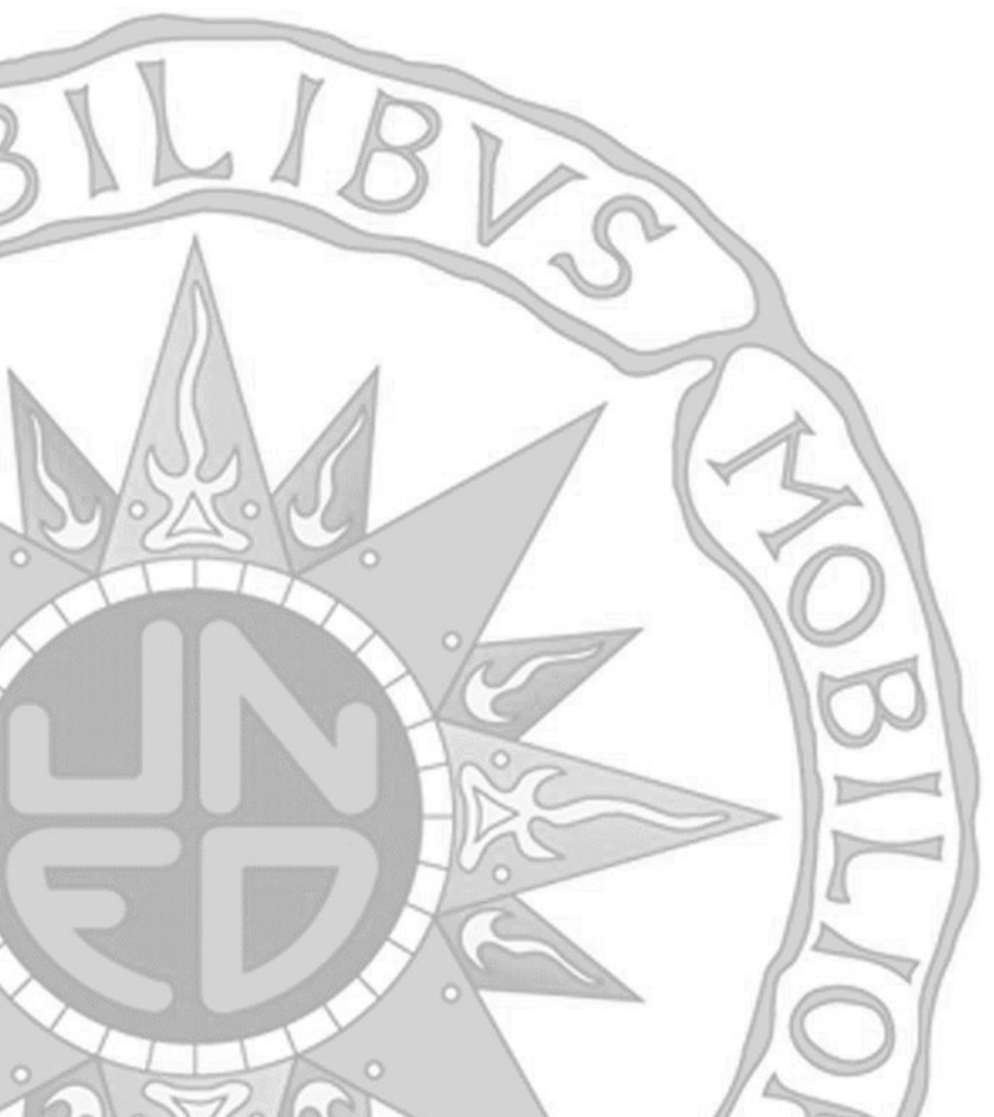

\author{
Universidad Nacional de Educación a Distancia (UNED) \\ Escuela Técnica Superior de Ingeniería Informática \\ Trabajo Fin de Máster \\ Máster Universitario en Inteligencia Artificial Avanzada
}





\section{Índice}

1. INTRODUCCIÓN

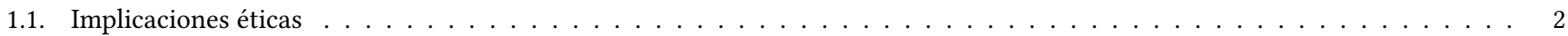

2. TRABAJOS RELACIONADOS 3

2.1. Ontologías, Minería de Textos y Razonamiento Basado en Casos . . . . . . . . . . . . . . . . . . . . . . 3

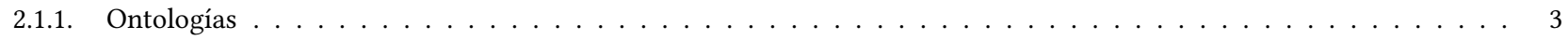

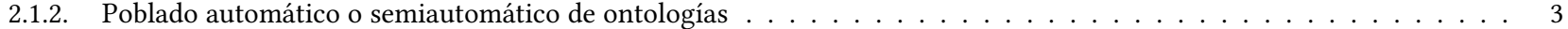

2.1.3. Razonamiento Basado en Casos y Minería de Textos. Combinación de métodos simbólicos y probabilistas . . . . . . 4

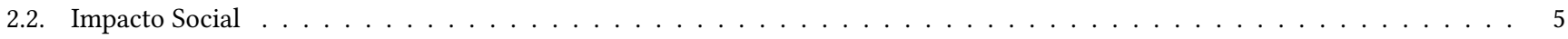

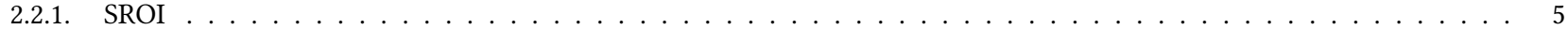

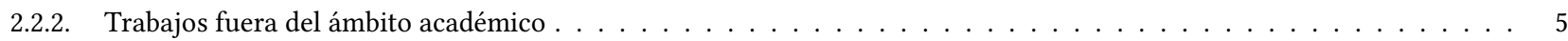

3. METODOLOGÍA DE INVESTIGACIÓN

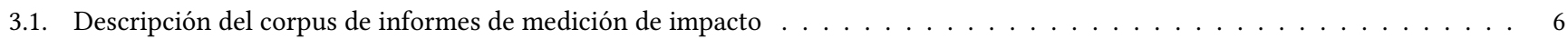

3.2. Fase I: Desarrollo de una ontología para el soporte de la minería de textos . . . . . . . . . . . . . . . . . . 6

3.3. Fase II: Procesamiento del corpus de informes de medición de impacto, y poblado de la ontología . . . . . . . . . . . . . . . 7

3.4. Fase III: Desarrollo de un prototipo de sistema de Razonamiento Basado en Casos para el soporte de la medición de impacto

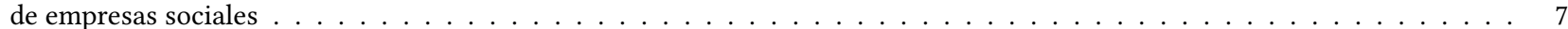

4. EXPERIMENTACIÓN, EVALUACIÓN Y ANÁLISIS DE RESULTADOS 7

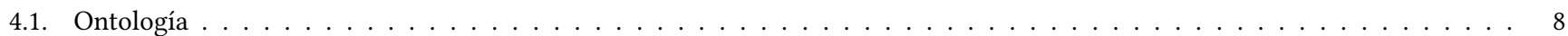

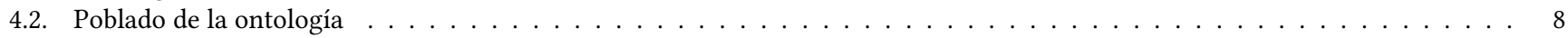

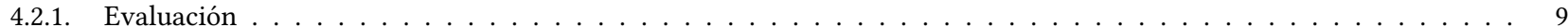

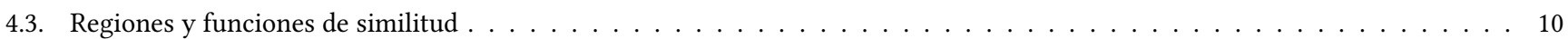

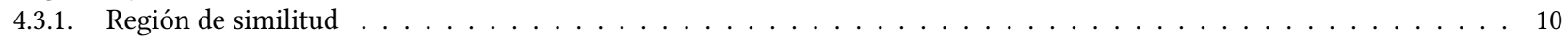

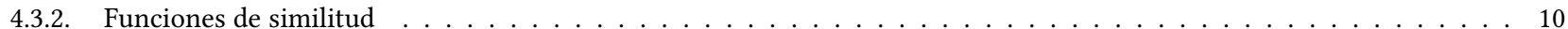

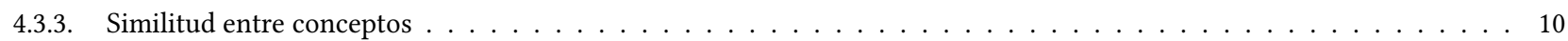

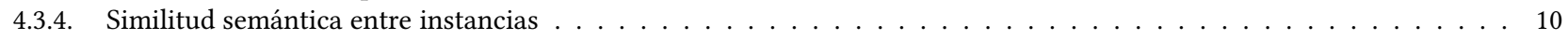

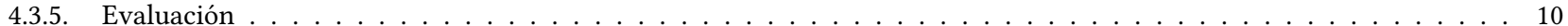

5. DETALLES SOBRE LA IMPLEMENTACIÓN 11

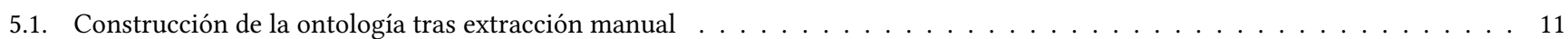

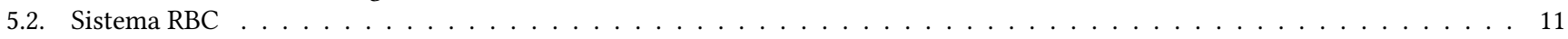

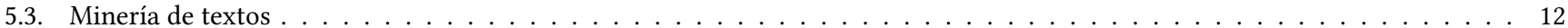

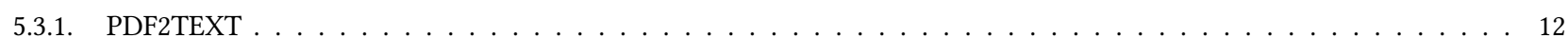

5.3.2. Autoetiquetado del corpus con los conceptos extraídos manualmente . . . . . . . . . . . . . . . . 12

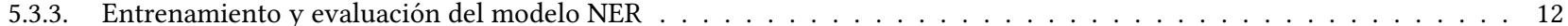

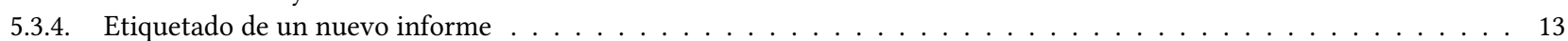

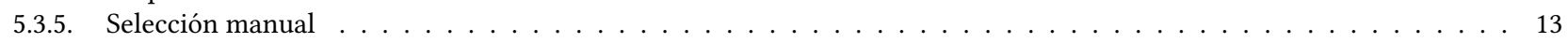

6. CONCLUSIONES 13

7. TRABAJOS FUTUROS 13

A. Tabla software para medición de impacto social identificado 16

B. Resultados completos función de similitud 19 

Abstract. Currently there is a trend that recovers the more traditional approaches to $\mathrm{AI}$ and investigates its combination with other techniques linked to Data Science, generating a symbiosis between symbolic and probabilistic methods that has aroused the interest of researchers and AI professionals. Through this research we delve into this field by proposing and studying a CBR system combined with text mining, applying an ontological approach in both tasks in a novel way, all applied to the measurement of social impact. Specifically, we define a RBC system model, we propose an ontology for the field of knowledge of social impact measurement and original semantic similarity functions for the recovery of cases.

In this article we report the results of our experimentation with SIAMES (Social Impact Advisor and Measurement System), a recommender system of social impact indicators. The system has two phases: first, it extracts structured information from a corpus of impact measurement reports using Semantic Text Mining based on ontologies. Second, taking inspiration from Case-Based Reasoning, it retrieves the most appropriate indicators for each social enterprise.

Resumen. Actualmente existe una tendencia que recupera los enfoques más tradicionales de la IA e investiga sobre su combinación con otras técnicas ligadas a la Ciencia de Datos, generando una simbiosis entre métodos simbólicos y probabilistas que ha despertado el interés de investigadores y profesionales de la IA. Mediante esta investigación profundizamos en este campo planteando y estudiando un sistema RBC combinado con minería de textos, aplicando de manera novedosa un enfoque ontológico en ambas tareas, todo ello aplicado a la medición de impacto social. En concreto, definimos un modelo de sistema RBC, proponemos una ontología para el campo de conocimiento de la medición de impacto social y funciones de similitud semántica originales para la recuperación de casos.

En este artículo reportamos los resultados de nuestra experimentación con SIAMES (Social Impact Advisor and Measurement System), un sistema recomendador de indicadores de impacto social. El sistema cuenta con dos fases: en primer lugar, extrae información estructurada de un corpus de informes de medición de impacto mediante Minería de Textos Semántica basada en ontologías. En segundo lugar, inspirándonos en el Razonamiento Basado en Casos, recupera los indicadores más adecuados para cada empresa social.

\section{INTRODUCCIÓN}

Durante el nacimiento y la primera expansión de la Inteligencia Artificial (IA), a partir de los años 50 del pasado siglo, los métodos simbólicos fueron el enfoque dominante. El modelo simbólico, también llamado representacional, utiliza símbolos (representaciones) del conocimiento para crear sistemas con un comportamiento inteligente, es decir, capaces de llevar a cabo tareas que, a priori, se consideran asociadas a la mente humana: razonar, aprender, buscar soluciones, etc. A este enfoque pertenecen, por ejemplo, los sistemas basados en reglas o el Razonamiento Basado en Casos (RBC).

Sin embargo, en las últimas décadas, ligado al espectacular crecimiento de la Ciencia de Datos, los métodos simbólicos fueron relegados a un segundo plano -considerándose, en cierto modo, anticuados- imponiéndose los métodos estadísticos como la vanguardia de la investigación. Los métodos probabilistas o estadísticos engloban diferentes enfoques muy en boga hoy en día: aprendizaje automático, minería de datos, redes neuronales, aprendizaje profundo, etc.

No obstante, actualmente existe una tendencia que recupera los enfoques más tradicionales de la IA, como los métodos simbólicos, e investiga sobre su combinación con otras técnicas ligadas a la Cien- cia de Datos [32] [23] [12], generando una simbiosis entre métodos simbólicos y probabilistas que ha despertado el interés de investigadores y profesionales de la IA. Dentro de esta corriente se está experimentando, por ejemplo, con modelos de RBC basados en Minería de Datos -en particular minería de textos incorporando técnicas de Procesamiento del Lenguaje Natural (PLN)- y en ontologías, y es en este campo donde se enmarca nuestra investigación.

Por otro lado, las tecnologías inteligentes ofrecen el potencial de generar niveles de prosperidad sin precedentes para todos, al tiempo que plantean crecientes desafíos en el ámbito ético, moral, legal, humanitario y sociopolítico. En este sentido, el informe de la ONU sobre pobreza extrema y derechos humanos recientemente publicado $^{1}$, advierte del riesgo de una distopía digital impulsora de una desigualdad creciente que está facilitando la creación de una vasta subclase digital. Este informe proporciona muchos ejemplos bien documentados en diferentes países sobre cómo tecnologías inteligentes deshumanizadas están creando barreras para acceder a una amplia gama de derechos sociales a quienes carecen de acceso a Internet y habilidades digitales.

Con este proyecto pretendemos -además de cubrir nuestro objetivo principal, que es la investigación sobre IA- facilitar la incorporación universal a los beneficios de la tecnología inteligente mediante su aplicación en el ámbito del emprendimiento social.

La medición del impacto social, si bien es un trabajo complejo, se puede realizar sobre cualquier proyecto del ámbito social facilitando información objetiva y veraz sobre los resultados de las diferentes actuaciones, mejorando de este modo el retorno social de las inversiones realizadas.

Consideraremos una Empresa Social como aquel agente de la Economía Social cuyo principal objetivo es tener un impacto social positivo por encima de la generación de beneficios para sus propietarios o accionistas. Funcionan en el mercado proporcionando bienes y servicios de manera empresarial e innovadora y reinvierten sus beneficios para generar un mayor valor social ${ }^{2}$.

Aunque las empresas sociales pueden abordar necesidades diferentes de las articuladas en los Objetivos de Desarrollo Sostenible (ODS) [29], se considera fuera de toda duda su aportación, directa o indirecta, para la consecución de los ODS [29] [41] [22] [38]. De hecho parecen ajustarse perfectamente al espíritu del ODS 19 Partnerships for the goals [29].

Las empresas sociales necesitan medir su impacto para satisfacer las necesidades internas de información, cumplir con las exigencias de los grupos de interés externos respecto a la rendición de cuentas sobre sus resultados y destacar el valor que generan [15] [36]. Para hacerlo, sin embargo, no se dispone de metodologías o unidades de medida aceptadas generalmente [15] [27].

La investigación llevada a cabo con SIAMES (Social Impact Advisor and Measurement System) tiene como aspiración facilitar la medición del impacto social, económico y medioambiental especialmente en el ámbito de la economía social, dotando a las empresas sociales de herramientas de valoración y comparación basadas en técnicas de Inteligencia Artificial. Este campo de trabajo, además de su interés por la contribución a la consecución de los ODS, presenta retos interesantes para la investigación en IA, tanto por el carácter pionero de nuestro trabajo como por las particularidades del dominio: la heterogeneidad de las fuentes de datos, la variedad de metodologías para la medición de impacto o la gran extensión, en número de palabras, de los conceptos con los que se trabaja en comparación

\footnotetext{
${ }^{1}$ https://www.ohchr.org/Documents/Issues/Poverty/

A_74_48037_AdvanceUneditedVersion.docx

2 https://ec.europa.eu/growth/sectors/social-economy/enterprises_en
} 
con la longitud habitual en otros ámbitos.

Con SIAMES experimentamos con la aplicación de métodos de minería de textos semántica basada en ontologías sobre un corpus de informes de medición de impacto para, posteriormente, utilizar los resultados en un sistema recomendador utilizando Razonamiento Basado en Casos. Por tanto, SIAMES, además de integrar técnicas simbólicas y de la ciencia de datos, hace énfasis en el valor de las técnicas de minería de datos de carácter semiautomático guiadas por conocimiento, es decir, aquellas basadas en modelos o usadas como procedimiento de destilación de datos para un posterior juicio humano.

En esta investigación definimos una ontología inicial y planteamos su poblado mediante minería de textos. Posteriormente, experimentamos con un sistema RBC para recuperar indicadores dado un nuevo proyecto de emprendimiento social concreto.

La ontología desarrollada en este trabajo es de especial importancia ya que se trata de la primera modelización formal del conocimiento realizada en el ámbito de la medición de impacto social hasta donde sabemos. Por lo tanto, esta definición no solo va a ser de utilidad para la presente investigación sino que puede constituir un punto de partida para diferentes trabajos posteriores en este ámbito.

Cabe destacar que la medición del impacto social no está limitada al ámbito de las empresas sociales. Existen varios actores que pueden tener la necesidad de medir el impacto social de sus actuaciones. Quizá el más claro sea el sector público, que, por supuesto, necesita buscar la eficiencia en sus actuaciones. Para asegurar el buen funcionamiento de las políticas que se lleven a cabo, el impacto de proyectos implementados directamente por la administración pública podría medirse sin demasiada dificultad adaptando los enfoques propuestos para la empresa social.

Por otro lado, las empresas tradicionales muestran una tendencia a integrar políticas de Responsabilidad Social Corporativa en sus procesos de gestión empresarial. Si bien esas medidas pueden ser controvertidas [5] [25], la necesaria medición del impacto [3][42] también podría plantearse en base a la investigación que aquí llevamos a cabo. De especial importancia, por la temática, es el sector de la Inteligencia Artificial, donde debido al actual debate sobre los riesgos éticos que comporta, se habla de integrar módulos de medición de impacto en las propias aplicaciones ${ }^{3}$.

Como comentábamos, la investigación realizada para el desarrollo de una ontología sobre la medición de impacto social que sirva de marco tanto para este como para futuros trabajos es de especial relevancia al no haber encontrado en nuestra búsqueda bibliográfica ninguna referencia en este ámbito; aunque, sin embargo, si encontramos algunos trabajos relacionados como [26] o [28].

En esta investigación planteamos como hipótesis la utilidad de un modelo de sistema RBC que combina métodos probabilistas y simbólicos, definido en la figura 2. En concreto, integramos la Minería de Textos Semántica con un enfoque ontológico y planteamos funciones de similitud semántica originales para la recuperación de casos. Para validarla, se ha construido un prototipo de aplicación que responde a dicho modelo y cuyos resultados han sido evaluados por Marta Solórzano, experta en el campo de la medición de impacto social. Además, validamos que este campo es realmente válido para la aplicación de nuestro modelo y mediante la experimentación se extraen conclusiones sobre otros posibles campos de aplicación.

Para finalizar esta introducción, incluimos unas reflexiones sobre las posibles implicaciones éticas del trabajo investigador y sus posi-

3 IEEE https://standards.ieee.org/content/ieee-standards/en/standard/70102020.html bles aplicaciones en el ámbito de SIAMES. En la sección 2, revisamos los estudios más relevantes para nuestra investigación publicados tanto en el ámbito de la medición de impacto social como de la minería de textos y los sistemas RBC. En la sección 3 describimos el plan de investigación, especificamos las fases en que van a llevarse a cabo y describimos en términos generales los experimentos y resto de tareas que van a llevarse a cabo en cada una de estas fases. En la sección 4 mostramos y evaluamos los resultados obtenidos experimentando con cada uno de los componentes del sistema propuesto como hipótesis. En la sección 5 describimos en detalle los pasos seguidos en el trabajo de investigación para implementar SIAMES, facilitando la replicabilidad de nuestros experimentos. En la sección 6 extraemos una serie de conclusiones en base a los resultados mostrados previamente. Por último, en la sección 7 dejamos marcadas las potenciales líneas de investigación futuras.

\subsection{Implicaciones éticas}

Como ya apuntábamos en la introducción, la ejecución de proyectos sociales, especialmente aquellos relacionados de un modo u otro con el ámbito empresarial, puede generar controversia. Por ejemplo, el sorprendente posicionamiento de algunas empresas, asimiladas por parte de la ciudadanía como poco éticas, en ciertas causas sociales ha acuñado términos como Pink washing ${ }^{4}$ o Greenwashing [11] en referencia a ese pretendido lavado de imagen.

Por otro lado, el propio emprendimiento social también cuenta con sus detractores. Es común la tendencia a dar por hecho el interés para el bien común de estas iniciativas; por ello conviene no perder el espíritu crítico para evitar perder, en consecuencia, el enfoque social. El artículo de Hyunbae Politics, Values and Social Entrepreneurship: A Critical Appraisal [8] expone los aspectos más polémicos en relación al emprendimiento social; es esencial conocerlos y tenerlos en mente cuando trabajamos en este ámbito.

SIAMES, al tratarse de una herramienta de apoyo a proyectos sociales sería también, inmediatamente y por razones obvias, socialmente positiva. Sin embargo, ¿qué ocurre en estos casos en los que el aspecto social de las iniciativas no está tan claro?

La visión subjetiva de los seres humanos nos permite en muchas ocasiones discernir si, bajo nuestra percepción, el objetivo tras una iniciativa que se autodenomine social repercute realmente y de manera positiva en el bienestar general. Las herramientas de Inteligencia Artificial orientadas a la medición del impacto social positivo, a no ser que cuenten con implementaciones especificas para ello, no van a ser capaces de evaluar de un modo tan abstracto el propósito real, pudiendo incluso favorecer aquellas iniciativas que cumplan ciertos criterios objetivos pese a estar muy lejos de tratarse de iniciativas realmente sociales.

Además de este tipo de conflictos, relacionados con actuaciones por parte de individuos o empresas realizadas de manera consciente y éticamente reprobables, nos encontramos con que la IA también puede incurrir en errores que provoquen situaciones injustas de forma impremeditada.

Por un lado, tenemos que, si bien la medición es crítica, la sobremedición puede llegar a ser contraproducente [35]. Además, en el caso de SIAMES, y en el de la medición de impacto en general, hay dos aspectos fundamentales a tener en cuenta. Por un lado los intangibles y por otro la medición del impacto con la moneda como unidad.

\footnotetext{
4 https://www.nytimes.com/2011/11/23/opinion/pinkwashing-and-israelsuse-of-gays-as-a-messaging-tool.html
} 
El uso de informes de medición reales hace que SIAMES vaya a utilizar en la mayoría de casos informes que utilizan el SROI como método para medir el impacto, debido a su fuerte implantación. Si bien el SROI tiene claramente aspectos positivos, el uso de proxies financieros puede traer consecuencias no deseadas como que proyectos que realmente tengan un alto impacto, pero que este se refiera a intangibles o que su monetización sea baja, queden relegados a posiciones secundarias cuando no debería ser así. De hecho, ya es conocido, en otros ámbitos, que la IA tiende a aumentar los sesgos humanos en vez de corregirlos si estos no son tenidos en cuenta [46].

Parece claro que la participación humana es capaz de obtener una visión global de la situación mientras que los sistemas automatizados pierden matices y aspectos que pueden ser clave a la hora de valorar la intencionalidad real de un proyecto. Por lo tanto, debemos tener especial cuidado con estos aspectos cuando planteamos la eliminación de las intervenciones humanas en los procesos que llevamos a cabo.

Es fundamental contar de manera transversal con estas reflexiones durante todo el desarrollo de la investigación y tratar de mitigar sus efectos negativos o, en caso de que no sea posible, exponer claramente las deficiencias que presenta nuestro sistema.

\section{TRABAJOS RELACIONADOS}

El presente artículo abarca una investigación multidisciplinar que incluye tanto la Medición de Impacto Social, por un lado, como la Minería de Textos Semántica, las Ontologías y el Razonamiento Basado en Casos por otro. Como adelantábamos en la introducción, nuestra investigación se centra en la combinación de métodos simbólicos y Ciencia de Datos, aprovechando las ventajas que nos ofrece cada uno de los enfoques basándonos en lo expuesto en la literatura detallada continuación. Además, consideramos de especial importancia nuestra aportación inédita en métricas de similitud semántica en ontologías del dominio del emprendimiento social.

\subsection{Ontologías, Minería de Textos y Razonamiento Basado en Casos}

La combinación de Minería de Textos y el Razonamiento Basado en Casos no es algo nuevo [43], [45] o [14]. Sin embargo, aportaremos un nuevo enfoque al aplicar ontologías para la minería de textos, así como la extracción de información con su uso posterior, no sólo para seleccionar los casos previos sino también para la resolución del problema. El papel de la ontología es fundamental en esta investigación, debido a que en el sector sobre el que planteamos nuestro estudio -la medición de impacto y el emprendimiento social- apenas se ha trabajado con ontologías.

Esta ontología será poblada de manera semiautomática mediante Minería de Textos Semántica, explorando las posibilidades que nos ofrece este enfoque para el poblado de ontologías. La Minería de Textos Semántica se refiere a los procesos de minería de datos que sistemáticamente incorporan conocimiento sobre el dominio en el proceso [13]. Por su parte, las ontologías son representaciones formales del conocimiento; éstas definen clases del dominio al que se refieran y sus relaciones entre ellas. El resultado de nuestra investigación es de especial importancia en el poblado semiautomático de ontologías debido a la heterogeneidad de los informes de medición de impacto existentes, lo que dificulta la tarea de extracción automática de información.
De lo que sí disponemos para nuestro trabajo es de un conjunto de informes de medición de impacto de calidad, por lo que experimentamos con el enfoque RBC. Los sistemas RBC se basan en recordar experiencias pasadas y usarlas para resolver situaciones actuales que son similares a las ya resueltas y almacenadas [17]. Como queda demostrado en las experiencias reportadas en la literatura científica, este enfoque se ajusta a nuestra casuística: disponemos de una serie de casos resueltos y bien documentados pero carecemos de procedimientos y teorías formalizados.

El objetivo al utilizar esta combinación de Minería de Textos y de Razonamiento Basado en Casos es tratar de maximizar los aciertos en la selección de casos previos. Por otro lado, el análisis semántico basado en ontologías trata de aprovechar las ventajas de este enfoque, como podemos ver en Dou [13].

En definitiva, presentamos una investigación original tanto por la aplicación de las diferentes tecnologías mencionadas en un campo poco explorado, como es la medición de impacto social para empresas sociales, como por la integración de todos estos enfoques para la resolución de un problema, algo por sí solo novedoso.

\subsubsection{Ontologías}

Las ontologías son representaciones semánticas del conocimiento capaces de ser utilizadas por sistemas informáticos; son un medio para, a través de definiciones formales y explícitas, poder compartir y reutilizar el conocimiento. Para que una ontología pueda ser considerada como tal, las conceptualizaciones que utilice deben ser comunes dentro de su ámbito de conocimiento; es decir, es necesario que exista consenso sobre ellas entre las personas relacionadas con su campo de conocimiento.

Tras una revisión bibliográfica identificando las ontologías ya existentes más relevantes para nuestro trabajo, encontramos dos de especial interés.

En primer lugar, Konys propone una ontología centrada en la sostenibilidad [26], entendiendo ésta, al igual que el emprendimiento social, en tres ámbitos principales: social, ambiental y económico. Se trata de una excelente definición de estructura, muy completa y amplia, en la que se inspira SIAMES para definir sus clases y relaciones. Sin embargo, aspectos fundamentales relevantes para nuestro trabajo (como indicadores o proxies), no existen en esta propuesta. Por otro lado, esta ontología se presenta sin poblar.

En segundo lugar, se ha tenido en cuenta la ontología propuesta por Li y otros [28]. Si bien, en este caso, el dominio no es el que nos ocupa, se trata de una definición ontológica de calidad en la que los indicadores cobran especial importancia. En concreto, en este trabajo se desarrolla una ontología de indicadores clave de rendimiento (Key Performance Indicator, KPI) para el ámbito de la gestión energética.

No se han encontrado en el estudio bibliográfico referencias a ontologías construidas para modelar el conocimiento en el ámbito que nos ocupa, las empresas sociales, pese a ser habituales las que pertenecen al ámbito empresarial en general.

\subsubsection{Poblado automático o semiautomático de ontologías}

Podemos distinguir diferentes tareas a realizar relacionadas con la extracción de información para la creación de ontologías. Algunas de estas tareas afectan a la estructura de la ontología mientras que, en otros casos, las tareas tratan de añadir instancias a una ontología con la estructura previamente definida. 
Las ontologías pueden ser definidas manualmente, a través del trabajo de expertos o mediante integración de ontologías existentes, o pueden ser construidas mediante procesos automáticos o semiautomáticos. A estos procesos se les conoce como Aprendizaje de Ontologías, Ontology learning en inglés. Por otro lado, cuando los procesos, en lugar de crear la ontología desde cero, modifican la estructura de una ontología existente (por ejemplo, añadiendo más clases y relaciones) hablamos de Enriquecimiento de la ontología o Ontology enrichment.

Sin embargo, cuando el procesado automático o semiautomático de fuentes para extraer conocimiento únicamente añade instancias a la ontología, sin modificar su estructura, es cuando lo denominamos Poblado de ontologías o Ontology population.

Para poder llevar a cabo estas tareas, necesitamos una o varias fuentes de datos de las que extraer la información. Estás fuentes pueden estar compuestas por información estructurada (entre otros, bases de datos), semiestructuradas (por ejemplo, en tablas dentro de documentos HTML) o sin estructurar, como en el caso de los corpora de textos en lenguaje natural.

En la bibliografía nos encontramos con varios enfoques a la hora de afrontar el poblado de ontologías utilizando como fuente un conjunto de textos sin estructurar. En algunos casos, centrados en resolver el problema para un dominio concreto -como la biomedicina [19] [40] o el turismo [39]- mientras que otros aportan una visión más generalista y buscan poder ser aplicados en cualquier campo del conocimiento [6] [33].

En los estudios de Petasis [37], Ruíz-Martínez [40] y Lubani [30] encontramos revisiones de los métodos más utilizados para afrontar el poblado de ontologías.

Tanto Ruíz-Martínez como Lubani distinguen subtareas dentro del proceso de poblado. Ambos coinciden en definir unas tareas principales, denominadas Initial requirements por Ruíz-Martínez y Information Extraction Engine en el caso de Lubani, que son las encargadas de extraer la conceptos y relaciones de las fuentes. Posteriormente, se realizan tareas para asegurar la consistencia, eliminar ambigüedades e incorporar los resultados definitivos en la ontología. En SIAMES planteamos, por el momento, un enfoque semiautomático, con intervención humana posterior, centrado únicamente en una de las tareas clave para el poblado de ontologías: la extracción de conceptos.

En numerosos trabajos, la extracción de información para el poblado de ontologías se ha separado tradicionalmente en dos tareas: por un lado, la extracción de las instancias que pasarán a formar parte de la ontología y por otro lado las relaciones entre dichas instancias [4] [30]. Observamos, a su vez, que existen dos enfoques ampliamente utilizados para abordar estas tareas de extracción de conocimiento: el Reconocimiento de Entidades Nombradas (Named Entity Recongnition, NER) y la definición de patrones lingüísticos. Nos encontramos, además, que estas técnicas también pueden ser utilizadas de manera combinada [9].

SIAMES centra su investigación en los apartados relativos a la extracción de instancias mediante técnicas PLN, en concreto, mediante NER, pero combinándolo con el enfoque RBC e integrando ambos enfoques.

\subsubsection{Razonamiento Basado en Casos y Minería de Textos. Combinación de métodos simbólicos y probabilistas}

El Razonamiento Basado en Casos en un mecanismo para resolver problemas basado en el pensamiento humano. Es habitual que las personas resolvamos problemas fijándonos en cómo se han re- suelto previamente problemas similares. Normalmente, un sistema $\mathrm{RBC}$ consta de cuatro fases, Recuperar, Reutilizar, Revisar y Retener, conocidas como las 4 Rs.

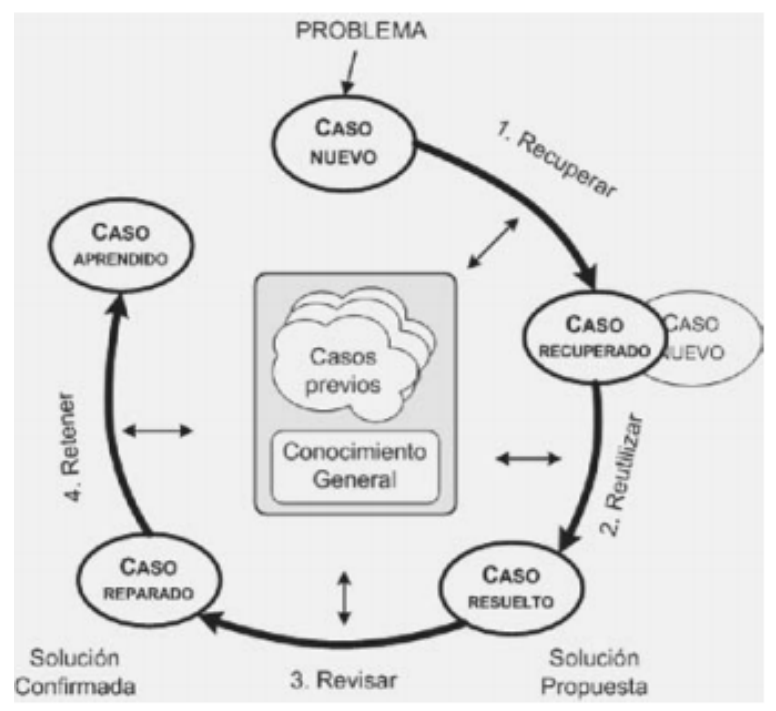

Figure 1. Ciclo RBC en Aamodt y Plaza [1]

La Minería de Textos, por su parte, trata de extraer información útil de una base de un conjunto de textos sin estructurar denominado corpus. Para ello utiliza diferentes técnicas de Procesamiento del Lenguaje Natural.

En la biografía encontramos varios usos combinados de Minería de Textos y Razonamiento Basado en Casos. En los trabajos analizados la Minería de Textos sirve, habitualmente, como medio para representar los casos y para recuperarlos. Así, en Shen [43] se define un marco de trabajo construido sobre estas dos técnicas. En resumen, en este trabajo se plantea el uso de la minería para extraer características (features) y palabras clave que sirvan posteriormente para modelar los casos.

En Xio [45] se plantea un sistema de minería de experiencias en el ámbito de la construcción sostenible (green building). En este caso, se hace uso de similitud semántica entre diferentes apartados relacionados con su ámbito de actuación para la recuperación de casos similares.

Por otro lado, en Dufour-Lussier [14] se busca lograr una representación rica de los casos de manera automática desde textos sin estructurar. Para ello, plantea los siguientes pasos: primero, realiza un procesado estándar de los textos mediante técnicas de PLN. Después, propone un método para resolver anáforas, por ejemplo, asociaciones de palabras del texto con objetos a los que se refiere. Por último, define la representación del caso basada en los resultados de los dos primeros procesos descritos.

Por su parte, He [21] utiliza tanto Minería de Textos como técnicas de la Web 2.0. En esta caso, plantea el uso de diferentes técnicas de PLN para clustering, extraer características y palabras clave y contabilizarlas. La principal diferencia en este caso es que el sistema de Minería de Textos sirve de apoyo para el constructor (humano) del sistema RBC, que debe saber interpretar la información que este sistema le proporciona para verificar y depurar sus resultados antes de integrarlos en el sistema RBC.

En el caso de SIAMES, como ya hemos adelantando, experimenta- 
mos con la extracción de entidades con el enfoque NER. El enfoque con el que hemos investigado se basa en los ya propuestos en la bibliografía, pero presentando dos particularidades. En primer lugar, los conceptos a extraer presentan diferencias notables con las entidades extraídas con los sistemas NER tradicionales (lugares, personas, organizaciones...), tanto en aspectos gramaticales como en la longitud de las mismas. Por ejemplo, no es extraño, esté definido por una frase de más de 20 palabras. En segundo lugar, además del enfoque RBC y la minería de textos, trabajamos con una ontología, adentrándonos en el estudio del sincretismo entre los enfoques originarios de la IA y la Ciencia de Datos.

\subsection{Impacto Social}

$\mathrm{Si}$ atendemos a las investigaciones realizadas hasta ahora que combinan IA y medición del Impacto Social, podemos observar que los estudios se centran o bien en el uso ético de la IA [31] [20] y su impacto [24] [10] o bien tratan de medir el impacto social , generalmente negativo, de diferentes actuaciones o fenómenos no relacionados con la implementación de proyectos sociales, como en estudios sobre desastres climatológicos [7] o sobre hábitos nocivos [44]. Sin embargo, el uso de la IA con el objetivo de ayudar a las empresas sociales en la medición de su impacto social positivo es un campo todavía por explorar en el que se enmarca este trabajo.

En general, la ambigüedad de las metodologías empleadas actualmente para medir el impacto social genera problemas. Existe por parte de los financiadores, tanto públicos como privados, una tendencia hacia la racionalización de los proyectos sociales [16] que ha aumentado la exigencia a las entidades ejecutoras de proyectos sociales de medidas formales de impacto social que proporcionen valores objetivos que puedan estandarizarse y verificarse. Y si bien existe un sinfín de métodos para medir el impacto social, la falta de convenciones sobre los indicadores a utilizar hace que el cálculo del valor social sea una tarea muy compleja y costosa [15] [36].

SIAMES está orientado a la estandarización de indicadores basando su sistema recomendador en informes de medición de impacto reales. De esta forma buscamos, en el largo plazo, obtener los mismos indicadores para proyectos sociales similares, facilitando la realización de comparativas entre diferentes proyectos y enfoques a la hora de resolver problemáticas sociales.

\subsubsection{SROI}

Pese a que el trabajo desarrollado en SIAMES puede aplicarse en casi cualquier enfoque de medición de impacto, hasta el momento los informes utilizados para la investigación se basan en el Retorno Social de la Inversión [34], SROI, por sus siglas en inglés, para el cálculo del impacto social.

El SROI es un sistema de medición del impacto social que surge como evolución del análisis de coste-beneficio. La principal característica del SROI es que implica poner un valor monetario a los beneficios y costos sociales, económicos y ambientales creados por una organización [2].

El uso de un enfoque financiero es el principal atractivo del SROI. Sin embargo, aunque se utilizan términos monetarios, el índice SROI no expresa valor financiero como tal, sino que debe verse como una forma integral de expresar la "moneda de valor social"[2]

\subsubsection{Trabajos fuera del ámbito académico}

Fuera del ámbito estrictamente académico, encontramos algunas aplicaciones que buscan facilitar la medición del impacto social y que pueden resultar de interés a la hora de experimentar con SIAMES. Se ha incluido una tabla resumen con las principales en el apéndice $\mathrm{A}$.

Vemos que básicamente cumplen varias funciones distintas: ayudan a crear el modelo para la medición del impacto, son útiles para la gestión, el monitoreo y la recopilación de datos y generan informes. En algunos casos, estas aplicaciones van orientadas para su manejo por el usuario final mientras que en otras ocasiones se centran en un usuario experto. Además, varias de ellas ofrecen servicios de consultoría asociados, lo que evidencia una vez más la dificultad de la medición del impacto social sin la intervención de un experto, campo en el que se centra SIAMES.

Entre los aspectos más interesantes para nuestro trabajo están las bases de datos de indicadores y cómo está estructurada la información. De la mayoría de ellos, en vista del funcionamiento de la aplicación, podemos inferir el modelo de datos fácilmente y en el caso de Global Value Exchange tenemos acceso al conjunto de datos, lo que nos ha servido de inspiración a la hora de modelar nuestra ontología.

Por último, alguna de ellas utiliza técnicas de Inteligencia Artificial, pero no con el mismo objetivo que en SIAMES. Por ejemplo, en Impact Social Vale Reporting se utiliza la IA para tratar de identificar impactos sociales en empresas tradicionales.

Mención aparte merece la compañía de reciente creación Clarity $^{5}$, que sí parece realizar tareas similares a las de SIAMES. Aunque no se centra en empreas sociales, trata de ayudar a medir el impacto social de las empresas empleando técnicas de IA. Sin embargo, apenas cuenta con documentación técnica o científica publicada, por lo que no ha resultado de mucha utilidad para nuestra investigación.

\section{METODOLOGÍA DE INVESTIGACIÓN}

Podemos dividir nuestro objetivo de investigación -la validación del sistema RBC propuesto con combinación de diferentes enfoquesen tres subojetivos que serán abordados en las distintas fases de la investigación.

En primer lugar, planteamos el diseño de una ontología, buscando validar que realmente el conocimiento del emprendimiento social relevante para la medición de impacto puede formalizarse en términos de una ontología.

En segundo lugar, acometemos el poblado de esta ontología, comprobando si es posible extraer de los informes la información estructurada necesaria para poblar la ontología. Conviene aclarar en este apartado que esta fase únicamente se ha cubierto a efectos ilustrativos y se ha complementado con un poblado manual para poder probar la hipótesis central.

Por último, experimentamos con funciones de similitud, tratando de formular una función que sirva para recuperar los casos relevantes.

Tanto para el primer como para el último apartado, contamos con la validación de los resultados por parte de una de las codirectoras de la investigación, Marta Solórzano, experta en el campo del emprendimiento social y la medición de su impacto.

La estrategia seguida en SIAMES para poder proveer a nuevos proyectos sociales de un conjunto de indicadores válidos para realizar la medición de su impacto social combina, fundamentalmente, técnicas de Minería de Textos y Razonamiento Basado en Casos.

Como se puede apreciar en la Figura 2, partimos de un corpus de informes (descrito en la siguiente sección) del que extraemos los in-

${ }^{5}$ https://clarity.ai 


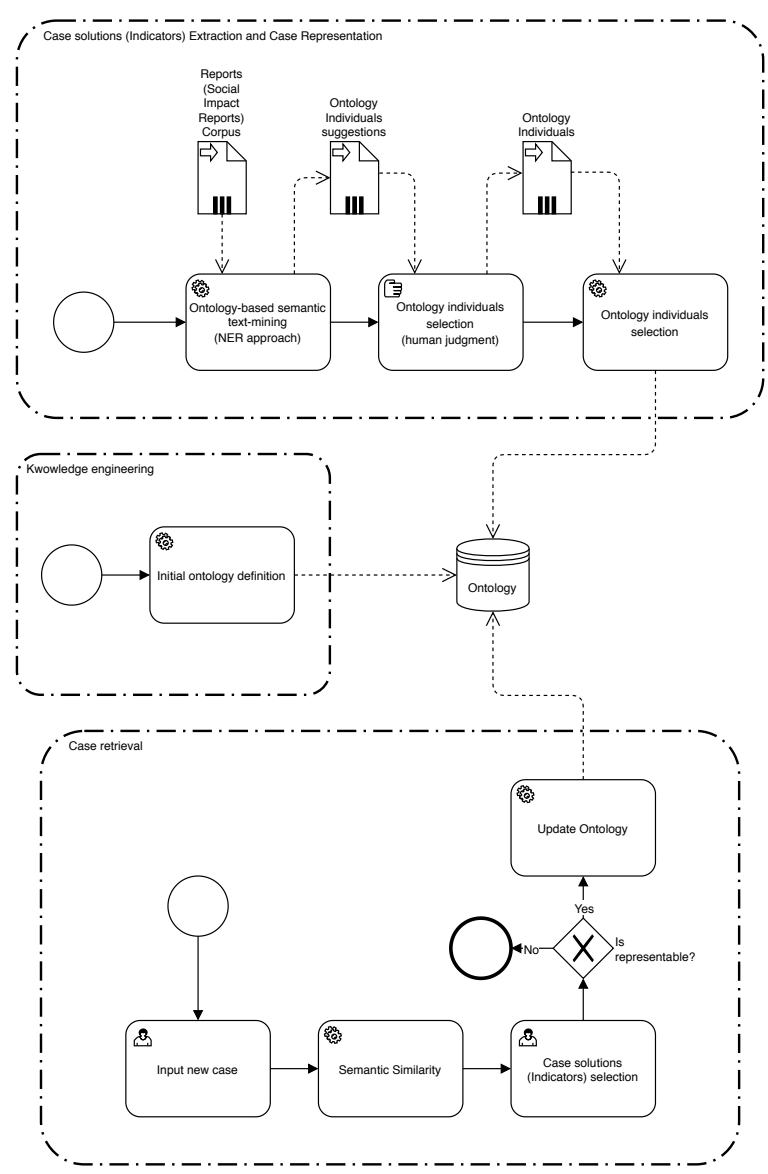

Figure 2. Modelo de RBC propuesto y experimentado con SIAMES dicadores para construir nuestra base de casos modelada como una ontología. Posteriormente, para recuperar los casos más similares al introducido por el usuario, comparamos los casos de nuestra base con el del usuario mediante una función de similitud semántica. Por último, siempre que fuera posible incluiríamos el nuevo caso en nuestra base de casos.

\subsection{Descripción del corpus de informes de medición de impacto}

Como primer paso para realizar nuestro estudio, recopilamos un corpus inicial compuesto por 380 informes de medición publicados libremente en Internet; aproximadamente la mitad de ellos procedentes de Social Value UK ${ }^{6}$. Sobre este corpus, se realizó primero una selección manual de los que mejores características reúnen para nuestro propósito: fundamentalmente, son aquellos que a nuestro parecer reflejan con la suficiente claridad los indicadores utilizados, valorando también positivamente aquellos que además incluyen proxies y/o fuentes de verificación. La mayoría de ello utilizan el SROI como método de medición de impacto.

Pese a que en este corpus, debido a la reducción realizada, el número de informes pueda parecer escaso para obtener resultados óptimos tras un proceso de minería de textos, se han considerado suficientes para realizar las investigaciones necesarias para plantear y validar nuestras hipótesis. No obstante, a futuro, está contemplada la incorporación de los informes que continúa recopilando Social Value $U K$ en su página web y a través de su proyecto $G V E^{7}$ ya que aportarán, sin lugar a dudas, mayor riqueza a nuestro corpus.

La dificultad para encontrar un número suficiente de informes para poder experimentar con métodos de extracción de información y la escasez de informes de calidad disponibles de manera libre explican también que el corpus esté conformado por documentos muy heterogéneos, tanto en su estructura y redacción como en las temáticas que trata.

\subsection{Fase I: Desarrollo de una ontología para el soporte de la minería de textos}

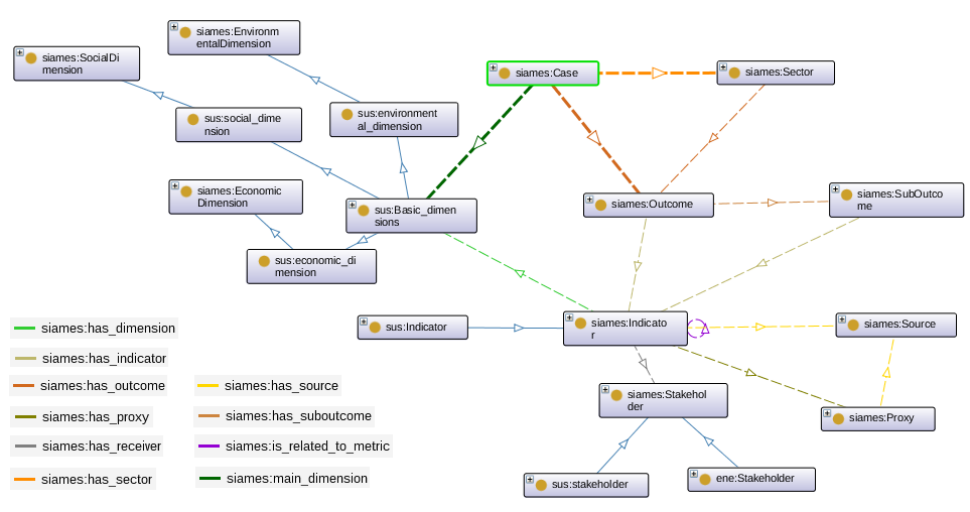

Figure 3. Estructura de la ontología de SIAMES. El prefijo siames: etiqueta nuestras clases originales, el prefijo sus: etiqueta clases de [26] y el prefijo ene: etiqueta clases de [28]

Tras la recopilación de informes, la siguiente fase a la que nos enfrentamos consiste en la definición y desarrollo de una ontología que

6 http://www.socialvalueuk.org/

7 http://www.globalvaluexchange.org/ 
sirva de soporte para el proceso de minería de textos posterior. La ontología constituye una parte fundamental de esta investigación, por lo que este apartado es crucial para el resultado final de nuestro trabajo.

Para la construcción de la ontología nos hemos basado, en lineas generales, en la metodología Methontology [18] por definir claramente los pasos a seguir para la construcción de ontologías y facilitar este trabajo. De acuerdo a lo sugerido en la metodología, hemos comenzado con la conceptualización de la ontología.

$\mathrm{Si}$ atendemos a conjuntos de indicadores existentes, como los indicadores recopilados por Bond para su herramienta Impact Builder ${ }^{8}$ o la base de datos IRIS de la Global Impact Investing Network ${ }^{9}$ podemos intuir varios aspectos que nos servirán para definir nuestra ontología. Si bien estos indicadores constituyen una magnífica recopilación, han sido elaborados en base a un estudio previo de varios informes dándoles una redacción que abarca en realidad varios posibles indicadores; por lo que no los encontramos exactamente como los encontraríamos en un informe de medición de impacto real. Sin embargo, su estudio nos resulta de interés para identificar clases de nuestra ontología.

Por ejemplo, en Bond, nos encontramos indicadores como \# and \% of children enrolled in [primary/secondary] school who are of [primary/secondary] school age. En primer lugar, lo normal sería que se midiera el impacto en una de las dos métricas, porcentaje o número, (o en las dos, pero por separado), por lo que en un informe de medición real lo normal sería encontrarnos indicadores como number of children enrolled in primary school who are of primary school age o percentage of children enrolled in secondary school who are of secondary school age. También, si atendemos por ejemplo al indicador \# and \% teachers who adapt lessons to the needs and abilities of learners in their classes (eg. learners with disabilities, language minorities, and traditionally excluded groups) vemos que se están englobando varios colectivos en el mismo indicador (también ocurría en el anterior). En un proyecto, lo habitual es que nos centremos en un colectivo concreto, por lo que la redacción de los indicadores en informes reales sería algo como number of teachers who adapt lessons to the needs and abilities of learners with disabilities in their classes o percentage of teachers who adapt lessons to the needs and abilities of ethnic minorities learners in their classes.

Además, nos hemos basado en las que consideramos que son las ontologías existentes que más relación tienen con nuestra investigación: las descritas en [26] y [28]. Cabe destacar, por resultar de especial interés, las dimensiones que marca [26] como básicas (social, ambiental y económica), así como el concepto de stakeholder definido en ambas.

En vista de lo expuesto hasta ahora, hemos optado por definir, por el momento, una ontología generalista definiendo tanto clases originales como clases basadas en las ontologías existentes citadas. En la Figura 3 se puede apreciar su estructura y sus relaciones con el resto de ontologías.

En resumen, esta ontología cumple con los requisitos que tenemos en esta investigación: abarca nuestro ámbito de trabajo, se basa en los estudios previos más relevantes para nuestra investigación, contiene información útil de los casos para poder recuperarlos y, por último, contiene los elementos clave que queremos mostrar: los indicadores de medición y, en caso de que estén disponibles, sus proxies y fuentes de datos asociadas.

\footnotetext{
8 https://www.bond.org.uk/resources/impact-builder

${ }^{9}$ https://iris.thegiin.org/
}

\subsection{Fase II: Procesamiento del corpus de informes de medición de impacto, $y$ poblado de la ontología}

La heterogeneidad de los informes de medición de impacto existentes ha sido el mayor escollo a superar en esta segunda fase.

El objetivo de esta fase de la investigación es extraer de los informes de medición los elementos necesarios para poblar nuestra ontología. La primera decisión, en vista del nivel de detalle elegido para la ontología y del corpus disponible, fue si tender a la automatización total o asumir una inevitable intervención humana.

Fundamentalmente, la falta de patrones fácilmente identificables en los informes de medición dificulta claramente la automatización total de esta tarea. Por lo tanto, se ha optado, por el momento, por un poblado semiautomático, basado en sugerencias del sistema pero con una revisión y selección definitiva de indicadores humana posterior. En concreto, nos centraremos en la extracción de conceptos del corpus de informes y dejaremos el resto de tareas (búsqueda de relaciones, desambiguación, consistencia, etc.) dependientes de la intervención humana.

Pese a que la investigación relacionada con técnicas PLN sería sin lugar a dudas interesante en este campo, no ha sido nuestro objetivo profundizar en ellas, sino que nuestros esfuerzos se han centrado en ilustrar su integración con otras técnicas de ontologías y con RBC.

\subsection{Fase III: Desarrollo de un prototipo de sistema de Razonamiento Basado en Casos para el soporte de la medición de impacto de empresas sociales}

En la tercera fase de nuestro desarrollo, construimos un prototipo de sistema de Razonamiento Basado en Casos. Si bien el objetivo de SIAMES en el medio plazo es poder servir de soporte para cualquier tipo de proyecto social, hemos decidido centrarnos en conseguir un prototipo funcional con una base de casos reducida por el momento, que podrá ser ampliada progresivamente.

Esta base de casos de proyectos reúne los casos ya modelados de acuerdo a la ontología y mediante las funciones de similitud creadas ex profeso para esta investigación, es capaz de recuperar los casos más similares al del usuario final comparando la representación del nuevo caso con la base de casos existente.

Con esta fase profundizamos en nuestra investigación sobre la combinación de diferentes enfoques, apoyando el proceso de minería de textos en el RBC y las métricas de similitud, en contraposición a otros enfoques, como podría ser el uso exclusivo de la minería de textos también en esta tarea.

\section{EXPERIMENTACIÓN, EVALUACIÓN Y ANÁLISIS DE RESULTADOS}

En este trabajo presentamos tres resultados de nuestra investigación: la ontología, poblada manualmente, un sistema para el poblado semiautomático, y una propuesta para la función de similitud definida en el espacio ontológico.

Para la evaluación de los resultados, tanto de la formalización de la ontología como de la selección de casos similares, se ha contando con la valoración de Marta Solórzano, investigadora de la empresa social y experta en el estudio sobre el empleo de herramientas de evaluación y monitorización del impacto social en la gestión empresarial. 


\subsection{Ontología}

La ontología es un importante resultado de la investigación. Se trata del primer modelo construido, hasta donde sabemos, para el ámbito del emprendimiento social y la medición de su impacto social.

Hemos construido la ontología basándonos en la estructura descrita en la Fase I. Esta ontología, se ha poblado manualmente con un conjunto reducido del corpus de informes.

La ontología contiene actualmente datos modelados de 14 informes de medición de impacto, con un total de 294 indicadores, 87 stakeholders, 287 otcomes, 17 sectores, 173 proxies y 141 fuentes. En la sección 5 se especifica como se ha llevado a cabo el poblado manual y en esta misma sección, en el apartado 4.2 se explica como e puede realizar el poblado semiautomático.

A continuación, vamos a describir cada uno de los componentes que conforman nuestra ontología, tanto clases como sus relaciones entre ellas y con las clases de las ontologías en las que nos hemos basado [26] o [28].

\section{- Case}

Representan cada uno de los casos que componen la ontología. Un caso es un proyecto de emprendimiento social, por lo que cada uno de los informes recopilados es un caso en nuestra ontología.

\section{- Indicator}

Los indicadores son uno de los elementos claves de la ontología. Se trata de referencias cuantitativas y cualitativas para evidenciar el avance en el cumplimiento de una actividad, output o resultado. Nos sirven para conocer si el cambio se ha producido y en que medida lo ha hecho

Son las representaciones que realmente nos ayudan a medir el Impacto Social de manera concreta. En nuestro sistema recuperador de casos, es el elemento que vamos a devolver. Ejemplos de indicadores podrían ser Number of young people now receiving a wage o Number of fathers who report having stopped negative coping strategies

\section{- Proxy}

Cuando utilizamos el SROI [34] para medir el impacto social, necesitmos incluir unos proxies financieron que nos ayuden a monetizar el indicador. Es decir, los proxies son las medidas de nuestros indicadores en términos económicos. Por ejemplo, para los indicadores anteriores tendríamos como proxies Tax and NI paid through new employment based on trainees salary y Wellbeing Value of relief from anxiety and depression.

\section{- Outcome y Suboutcome}

Son los resultados de cambio que esperamos que tenga el proyecto social. El efecto (cambio) social, tanto a largo como a corto plazo, logrado en beneficio de la población objetivo como resultado de la actividad emprendida a fin de lograr cambio social y teniendo en cuenta cambios positivos y negativos ${ }^{10}$. En ocaciones, un resultado amplio puede dividirse en varios para failitar las tareas de seguimiento del proyecto en general y de medición del impacto en particular.

\section{- Sector}

Es el ámbito de trabajo, en el aspecto social, de la empresa. Ejemplos de sectores pueden ser health, inmigration o culture.

\section{- Source}

Es la fuente de donde vamos a tomar las mediciones para indicadores y proxies. Puede ser cualquier tipo de documento en el que,

\footnotetext{
${ }^{10} \mathrm{https} / /$ ec.europa.eu/social/BlobServlet?docId=13401\&langId=en
}

de algún modo, podamos consultar los datos necesarios para calcular los valores que tenemos que asignar, por ejemplo, pueden ser desde encuestas hasta datos oficiales del paro de una determinada región.

\section{- Stakeholder}

Puede ser cualquiera de las partes (grupos de personas o instituciones) a las que afectan o pueden afectar los resultados e impactos del proyecto. En la ontología, cada uno de estos grupos de interés tiene sus propios indicadores de medición de impacto.

- BasicDimension: SocialDimension, EconomicDimension, EnvironmentalDimension

Son las tres principales dimensiones que vamos a medir, que coinciden con las dimensiones fundamentales entre las que un emprendimiento social debe buscar un equilibrio.

\section{- has_dimension}

Relaciona un indicador con una o varias de las dimensiones básicas en función de si este indicador trata de medir una o varias de ellas.

\section{- main_dimension}

Relaciona un caso con una de las dimensiones básicas, estableciéndose como principal.

\section{- has outcome}

Relaciona un sector con los resultados que se refieren a él.

- has_suboutcome

Relaciona un resultados con sus resultados dependientes, si los tuviera.

\section{- has_sector}

Relaciona un caso con sus sectores principales.

- has_proxy

Relaciona un indicador con su/s proxy/ies financieros.

- has_source

Relaciona tanto indicadores como proxies con sus fuentes.

- is_related_to_metric

Relaciona indicadores entre sí, si tienen algún tipo de similitud.

\section{- has_literal}

Es una propiedad que almacena el literal del concepto, es decir, el texto tal cuál aparece en el corpus.

\subsection{Poblado de la ontología}

El poblado de la ontología se realiza de manera semiautomática: para un nuevo informe, el sistema nos devuelve los términos susceptibles de formar parte de la ontología y, mediante intervención humana, se depuran y relacionan antes de ser incluidos en la ontología.

Este enfoque está basado en los que ya hemos visto en los trabajos relacionados. En nuestro caso, por el momento, únicamente tratamos de resolver uno de los problemas, que es la extracción de características de nuestro corpus para el posterior poblado de la ontología.

Hemos experimentado con el enfoque NER. Para ello, en primer lugar, y basándonos en las extracciones manuales que hemos realizado, hemos recopilado y formateado un pequeño conjunto de datos que nos permite entrenar un sistema NER para la extracción de indicadores, sectores, resultados, fuentes y proxies.

Por la simpleza de su uso y la calidad de los resultados de evaluación, hemos decidido utilizar spacy ${ }^{11}$ para estas tareas. Partimos de un corpus de informes sin etiquetar, pero con sus conceptos extraídos manualmente y recogidos en nuestra ontología. Por lo tanto,

${ }^{11}$ https://spacy.io) 
primero hemos etiquetado el corpus, lo hemos dividido en tres archivos (train, test y eval) y hemos entrenado y evaluado el modelo. La información detallada sobre este proceso se encuentra en la sección 5.

En la imagen 4 se pueden ver algunos ejemplos con los resultados de aplicar el modelo al corpus de informes.

$10 \$ 11,400,000.000 .26 \$ 2,964,000.00$ surveys and interviews

SOURCES ; secondary source materials ; citation [20 ] from

assumption tab government agencies STAKEHOLDERS enhances coastal protection for adjacent STAKEHOLDERS communities

savings INDICATORS on storm protection ( \$ /acre / year) $10 \$$

$619.00 /$ acre / year

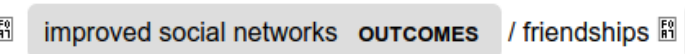
improved social networks outcomes / friendships 蜸 better

physical health OUTCOMES Fin stronger social networks child

participant STAKEHOLDERS weekly visit to allotment during term

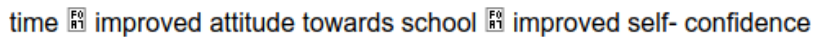

卧 improved performance in school 臤 higher academic

achievement volunteer STAKEHOLDERS facilitate and assist

allotment sessions

Figure 4. Salida del modelo NER

\subsubsection{Evaluación}

Para evaluar el sistema NER se han utilizado las métricas Precision (NER P), Recall (NER R) y F-score (NER F). Para calcular las métricas nos basamos en la siguiente matriz de confusión:

\begin{tabular}{|c|c|c|c|}
\cline { 3 - 4 } \multicolumn{2}{c|}{} & \multicolumn{2}{c|}{ Actual label } \\
\cline { 3 - 4 } \multicolumn{2}{c|}{} & True & False \\
\hline \multirow{2}{*}{ Predicted label } & True & TP & FP \\
\cline { 2 - 4 } & False & FN & TN \\
\hline
\end{tabular}

Siguiendo la tabla, queda perfectamente descrito a qué nos referimos con positivo correcto (True Positive (TP), falso positivo False Positive (FP), negativo correcto True Negative (TN) y falso negativo False NEgative (FN).

En base a estos términos, definimos las métricas como sigue:

\section{- NER P}

$$
\text { Precision }=\frac{T P}{T P+F P}
$$

\section{- NER R}

$$
\text { Recall }=\frac{T P}{T P+F N}
$$

- NER F

$$
F-\text { score }=\frac{2 * \text { Precision } * \text { Recall }}{\text { Precision }+ \text { Recall }}
$$

Los resultados del sistema de extracción (NER) para todas las entidades son los reflejados en la tabla 1.

\begin{tabular}{|l|l|}
\hline NER P & 91.02 \\
\hline NER R & 53.09 \\
\hline NER F & 67.06 \\
\hline
\end{tabular}

Table 1. Resultados generales de la extracción

Los resultados para cada tipo de entidad se muestran en las tablas $2,3,4,5$ y 6 .

\begin{tabular}{|l|l|}
\hline NER P & 81.52 \\
\hline NER R & 48.06 \\
\hline NER F & 60.47 \\
\hline
\end{tabular}

Table 2. Resultados para Outcomes

\begin{tabular}{|l|l|}
\hline NER P & 78.50 \\
\hline NER R & 47.12 \\
\hline NER F & 58.89 \\
\hline
\end{tabular}

Table 3. Resultados para Stakeholders

\begin{tabular}{|l|l|}
\hline NER P & 80.53 \\
\hline NER R & 47.12 \\
\hline NER F & 58.89 \\
\hline
\end{tabular}

Table 4. Resultados para Sectors

\begin{tabular}{|l|l|}
\hline NER P & 94.91 \\
\hline NER R & 96.31 \\
\hline NER F & 95.60 \\
\hline
\end{tabular}

Table 5. Resultados para Sources

\begin{tabular}{|l|l|}
\hline NER P & 94.12 \\
\hline NER R & 9.04 \\
\hline NER F & 16.49 \\
\hline
\end{tabular}

Table 6. Resultados para Indicators 


\subsection{Regiones $y$ funciones de similitud}

\subsubsection{Región de similitud}

Definiendo regiones de similitud tratamos de delimitar un subconjunto dentro de nuestra ontología sobre el que aplicar la función de similitud. En nuestro caso, recortar la ontología en exceso mediante regiones podría mermar el éxito de nuestro sistema: en realidad, dos proyectos con ciertas similitudes y pese a sus diferencias pueden compartir sus indicadores.

Por lo tanto, solo vamos a definir una región de similitud que sirva de filtro a nuestra ontología: la dimensión principal de cada caso. Por ejemplo, si nos encontramos con un caso cuya dimensión principal sea la social, eliminaremos de nuestro subconjunto los casos con dimensión ambiental o económica.

\subsubsection{Funciones de similitud}

Por otro lado, definiremos nuestra función de similitud entre dos casos $C_{1}, C_{2}$ como:

$$
\operatorname{Sim}\left(C_{1}, C_{2}\right)=\frac{\sum_{i=1}^{k} \operatorname{Sim}\left(c_{i}^{k}, c_{j}^{k}\right)}{k}
$$

Es decir, la media de las similitudes de los conceptos comparables. Las características comparables a tener en cuenta, de acuerdo a nuestro modelo ontológico, serán las instancias de los conceptos: Sectors, Outcomes, Suboutcomes and Stakeholders.

\subsubsection{Similitud entre conceptos}

En nuestro modelo, consideramos que para cada uno de estos conceptos tendremos una o varias instancias. De este modo, supongamos que, por ejemplo, un caso $C 1$ tiene dos sectores asociados, pongamos Sexual Health y youth, mientras que otro caso $C 2$ tiene tres, que pueden ser gender, young y sexual health. A primera vista podemos ver que ambos casos cuentan con dos sectores casi idénticos y que el segundo caso tiene un sector más con menor similitud.

Para comparar el concepto Sectors, aplicando similitud semántica, de tal forma que podamos puntuar positivamente las coincidencias y restar a la puntuación un valor razonable por aquellas instancias menos similares, hemos decidido aplicar la siguiente fórmula:

$$
\operatorname{Sim}\left(C_{1 S}, C_{2 S}\right)=\operatorname{Sim}\left(c_{1}^{S}, c_{2}^{S}\right)=\frac{\sum_{i=1}^{k} \max \left(\operatorname{Sim}\left(i_{1 S}^{k}, i_{2 S}^{1 . . n}\right)\right)}{k}
$$

Que define la similitud del concepto Sectors, representado con $S$, entre los casos $C 1$ y $C 2$ siendo $k$ el número de instancias del concepto sectores que tiene el caso 1 y $n$ el número de instancias del concepto sectores que tiene el caso dos. Es decir, comparamos cada instancia del concepto sectores del caso 1 con todas las instancias del concepto sectores del caso 2, seleccionando la más similar. Una vez tenemos un valor de similitud para cada instancia del caso 1 , calculamos la media de similitudes para obtener la similitud entre los casos para el concepto Sectors.

En nuestro ejemplo, para calcular $\operatorname{Sim}\left(C_{1 S}, C_{2 S}\right)$, comenzamos, por ejemplo, con la instancia Sexual health. Comparamos esta instancia con las otras tres del caso 2, gender, young y sexual health. La más similar, con un valor de 1 al ser idénticas, será la tercera. Después, buscamos la similitud para la segunda instancia, youth que, en este caso, todo parece indicar que el valor más alto lo obtengamos al compararlo con young. Y, teniendo ya los dos valores de similitud, simplemente calculamos la media para obtener el valor global.

Cabe destacar que, si realizamos la comparación a la inversa, es decir, el caso 2 con el caso 1, obtendremos valores distintos, ya que, en este caso, tenemos tres instancias que comparar. Para las dos últimas, young y sexual health, obtendremos los mismos valores. Sin embargo, cabe esperar que obtengamos un valor más bajo para gender, que será el valor de similitud más alto al compararlo con young y sexual health. Esta similitud, al calcular la similitud del concepto obteniendo la media, bajará el valor.

Pese a que pueda parecer algo extraño, hemos considerado que es un efecto deseable: si queremos recuperar indicadores para el caso 1 vamos a poder encontrarlos en el caso 2 , ya que cubre sus mismos sectores (y uno más), pero, si lo que queremos es obtener indicadores para el caso 2, el caso 1 solo nos proporcionará parte y, es posible, que tengamos algún otro caso en nuestra base de casos que cubra mejor los sectores del caso 2 .

Seguiremos este mismo enfoque en todos los conceptos.

\subsubsection{Similitud semántica entre instancias}

Para calcular la similitud entre instancias, utilizamos el algoritmo word2 $\mathrm{vec}^{12}$, un modelo de tipo word embedding ampliamente utilizado en este tipo de tareas, y compararemos los vectores utilizando el coseno de similitud

$$
\operatorname{Sim}=\frac{A B}{\|A\|\|\mid B\|}
$$

Esta función devuelve un valor en el rango $[0,1]$. Por lo tanto, la similitud entre dos casos, siendo la media de la similitud entre características, será también un valor en el rango [0,1].

En este primer enfoque, para cada nuevo caso, buscaremos los casos más similares y, una vez localizados, extraeremos sus indicadores, proxies y fuentes relacionadas de la ontología (una tarea trivial ya que los casos están previamente modelados). Por úlitmo, los usuarios de SIAMES serán los encargados de seleccionar cuáles de los indicadores sugeridos por el sistema quieren usan en sus proyectos. En el futuro, se pueden refinar los resultados, por ejemplo, seleccionando los indicadores más similares de entre los indicadores de los casos más similares.

\subsubsection{Evaluación}

Para evaluar las funciones de similitud de manera empírica, se han realizado experimentos cruzando los 14 casos entre sí (sin compararlos consigo mismo) y recuperando un máximo de tres casos similares para cada uno de ellos.

De nuestros 14 casos para experimentar, 12 pertenecen al mismo sector principal (social) mientras que otros 2 perteneces a otro sector (ambiental). Por lo tanto, para cada uno de los 12 primeros casos vamos a obtener como resultado los tres más similares y en el los otros dos casos solo obtendremos un caso recuperado (el único disponible, teniendo en cuenta que son sólo dos).

Para evaluar los resultados se han tenido en cuenta dos métricas: la precisión y el fall-out. Los casos relevantes y no relevantes se corresponden con los que, en base al conocimiento del campo a tratar, se han seleccionado de manera subjetiva por el autor y las codirectoras de este trabajo.

\section{Precisión}

12 https://code.google.com/archive/p/word2vec/ 
Con la precisión medimos la fracción de casos recuperados que son realmente relevantes para el usuario. Viene definida por la función:

$$
\text { Precision }=\frac{\text { Casos relevantes recuperados }}{\text { Casos recuperados }}
$$

La precisión vendrá definida, por tanto por, un valor entre 0 y 1 , siendo mejores los resultados cuanto más se aproxime a 1.

\section{Fall-out}

Con esta métrica calculamos la proporción de errores en nuestro resultados y la definimos como:

$$
\text { Fall }- \text { out }=\frac{\text { Casos no relevantes recuperados }}{\text { Casos no relevantes }}
$$

$\mathrm{Al}$ igual que en la precisión, el fall-out vendrá definido por un valor entre 0 y 1 , pero en este caso cuanto más próximo sea a 0 mejores serán nuestros resultados.

\section{Resultados}

En base a los resultados de similitud obtenidos, expuestos en el apéndice $\mathrm{B}$, obtenemos los valores que mostramos en las tablas 7. y

\begin{tabular}{|c|c|c|c|}
\hline Caso & $\begin{array}{c}\text { Casos relevantes } \\
\text { recuperados }\end{array}$ & $\begin{array}{c}\text { Casos no relevantes } \\
\text { recuperados }\end{array}$ & $\begin{array}{c}\text { Casos relevantes } \\
\text { no recuperados }\end{array}$ \\
\hline 001 & 3 & 0 & 0 \\
\hline 002 & 2 & 1 & 1 \\
\hline 003 & 1 & 2 & 2 \\
\hline 004 & 3 & 0 & 0 \\
\hline 005 & 1 & 0 & 1 \\
\hline 006 & 2 & 1 & 0 \\
\hline 007 & 3 & 0 & 1 \\
\hline 008 & 2 & 1 & 0 \\
\hline 009 & 3 & 0 & 0 \\
\hline 010 & 1 & 0 & 0 \\
\hline 011 & 3 & 0 & 1 \\
\hline 012 & 2 & 1 & 1 \\
\hline 013 & 2 & 1 & 0 \\
\hline 014 & 3 & 0 & \\
\hline
\end{tabular}

Table 7. Casos recuperados con funciones de similitud

\begin{tabular}{|l|l|}
\hline Precisión & 0.8421 \\
\hline Fall-out & 0.0556 \\
\hline
\end{tabular}

Table 8. Resultados funciones de similitud

\section{DETALLES SOBRE LA IMPLEMENTACIÓN}

Todo el código utilizado para realizar esta investigación está liberado como software libre en GitLab ${ }^{13}$. El código se ha desarrollado únicamente con propósitos investigadores, lo que quiere decir que se pueden realizar bastante mejoras y optimizaciones en la arquitectura y en el propio código. Sin embargo, se ha procurado que sea relativamente sencillo de utilizar para garantizar la replicabilidad de nuestro trabajo.

\footnotetext{
13 https://gitlab.com/siames
}

Los informes utilizados están también disponibles ${ }^{14}$. Por favor, tenga en cuenta que, aunque los informes están publicados libremente en la paǵina web de Social Value UK, pueden tener derechos de autor. Solamente se redistribuyen con fines de investigación, se debe contactar con los propietarios para otro tipo de usos.

El código se ha desarrollado en Python y se han utilizado varias librerías y proyectos de terceros. Principalmente, se ha utilizado ghostscript ${ }^{15}$ para preprocesar los PDFs, Parsr $^{16}$ para convertir los PDF a texto, Spacy para la mayoría de tareas relacionadas con el Procesamiento del Lenguaje Natural y OWLready $2{ }^{17}$ para trabajar con la ontología.

\subsection{Construcción de la ontología tras extracción manual}

Para la construcción y el poblado manual de la ontología, necesario tanto para validar las funciones de similitud como para entrenar el modelo NER para el poblado semiautomático, se han seguido los siguientes pasos.

En primer lugar, se han seleccionado 14 informes para la realización de los experimentos. La selección se ha hecho en base a la calidad de los informes y a la claridad a la hora de mostrar los datos de nuestro interés. La extracción se ha realizado directamente desde los PDFs, sin procesar. Para un humano, resulta más sencillo localizar los indicadores de esta forma que si hubiéramos convertido los PDFs a texto plano.

Posteriormente, se ha rellenado un fichero CSV con los datos ${ }^{18}$, a partir del cuál nos va a resultar muy sencillo construir la ontología en cualquier formato estandarizado.

Para leer este CSV y construir la ontología, en nuestro caso en formato OWL, hemos utilizado pandas y OWLready2. El script de generación se puede consultar en el repositorio de GitLab.

\subsection{Sistema RBC}

El sistema RBC para nuestra experimentación consta fundamentalmente de dos apartados. En primer lugar, calculamos y almacenamos los vectores para todas las características de la ontología. En segundo lugar, comparamos los casos entre sí para evaluar nuestro sistema.

Para la vectorización, se ha utilizado Spacy. Un vector es una representación de las palabras que componen el concepto, conformado por número en coma flotante, de este estilo:

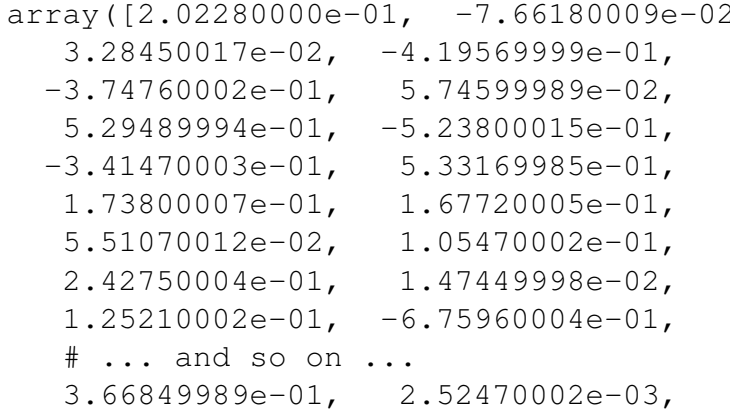

${ }^{14}$ https://drive.google.com/drive/folders/

1heBLDlaoSynxObhMr6M9eCwf0FzioFar?usp=sharing

15 https://www.ghostscript.com/

$16 \mathrm{https} / / /$ github.com/axa-group/Parsr

17 https://pypi.org/project/Owlready2/

18 Ver el repositorio de GitLab para consultar el archivo 


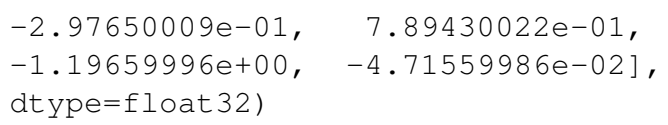

En nuestro caso, hemos utilizado el modelo de spacy en_core_web_lg, compuesto por 685000 vectores únicos de 300 dimensiones y entrenado con los corpora OntoNotes $5^{19}$ y GloVe Common $\mathrm{Crawl}^{20}$.

El cálculo de la similitud se ha realizado apoyándose también en la librería spacy y de acuerdo a las funciones similitud descritas en la sección 4 .

El código completo puede consultarse en el repositorio vectorize de GitLab.

\subsection{Minería de textos}

El proceso de minería de textos conlleva varias fases representadas en el esquema de la figura 5

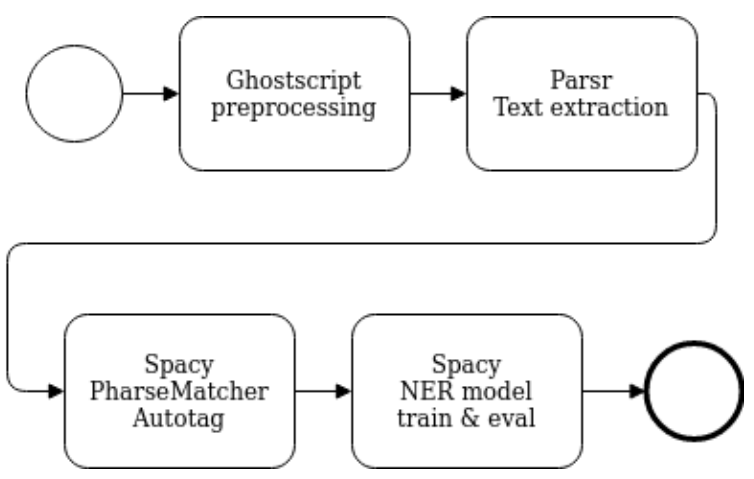

Figure 5. Proceso para la minería de textos

\subsubsection{PDF2TEXT}

En primer lugar, hemos tenido que convertir los informes, publicados en PDF, a texto plano para poder trabajar con ellos. La principal dificultad en este punto ha sido la correcta extracción de la información contenida en tablas incustradas en los PDFs, vital para nuestro caso ya que buena parte de las características a recuperar se encuentran en ellas.

Al tratarse de una tarea común y ampliamente abordada, sencillamente hemos utilizado las herramientas que mejores resultados nos han ofrecido de entre las diversas opciones.

En primer lugar, se han detectado problemas al leer tablas con colores de fondo. Utilizando ghostscript con los parámetros indicados abajo para convertir el documento a blanco y negro se ha logrado que la lectura de las tablas problemáticas quedara resuelta.

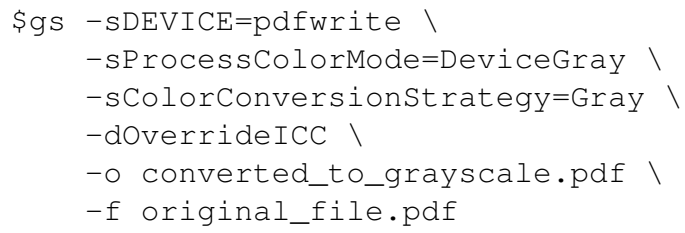

19 https://catalog.ldc.upenn.edu/LDC2013T19

20 https://nlp.stanford.edu/projects/glove/
Después, hemos procesado los PDFs resultantes en escala de grises con Parsr. Se ha optado por pdfjs como extractor de texto y $\mathrm{Ca}-$ melot con el método latice para extraer el contenido de las tablas, obteniendo un resultado bastante aceptable para nuestro propósito en todos los casos.

\subsubsection{Autoetiquetado del corpus con los conceptos extraídos manualmente}

Una vez tenemos el corpus es texto plano, es necesario etiquetarlo indicando las ocurrencias de cada uno de los individuos de nuestra ontología. Por rapidez y eficacia, como ya hemos visto anteriormente, la extracción manual de características se ha realizado directamente desde los PDFs, ya que con el texto formateado son mucho más fáciles de localizar.

Como ya tenemos en nuestra ontología los diferentes individuos, solo es necesario buscarlos dentro del corpus para añadirles las etiquetas correspondientes y generar los ficheros necesarios para el entrenamiento del modelo NER. Para esta tarea, hemos utilizado Spacy PhraseMatcher $^{21}$, un matcher basado en reglas.

Este proceso, aunque no perfecto, nos ha permitido etiquetar todo el corpus en un breve período de tiempo y sin intervención humana. El código utilizado en este apartado se encuentra en el fichero tagger.py del repositorio.

\subsubsection{Entrenamiento y evaluación del modelo NER}

Como ya hemos introducido en la sección sobre trabajos relacionados, tenemos varias opciones para construir nuestro modelo. Por el momento, profundizar en este aspecto excede de los objetivos de este trabajo, ya que hemos buscado validar el concepto completo del sistema, integrando enfoques simbólicos y estadísticos, con un prototipo, por lo que se ha optado por una solución rápida, sencilla de implementar y con unos resultados que, aunque claramente mejorables, hemos considerado suficientes para nuestros propósitos.

Tanto para el entrenamiento del modelo, como para la evaluación y empaquetado del mismo se ha utilizado la librería Spacy. En concreto, los cuatro comandos mostrados a continuación han sido suficientes para la ejecución de las tres tareas mencionadas precedidas por una conversión de formato.

\section{Conversión de formato de los archivos}

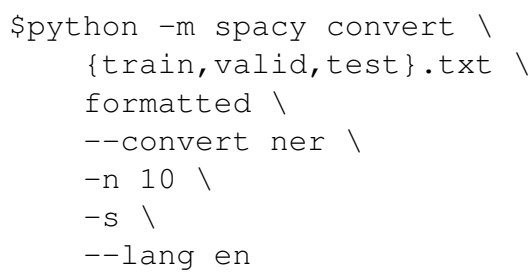

\section{Entrenamiento del modelo}

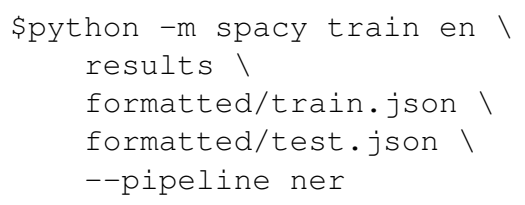

\section{Evaluación del modelo}

${ }^{21}$ https://spacy.io/usage/rule-based-matching\#phrasematcher 


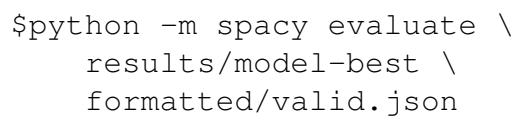

\section{Empaquetado del modelo para su uso posterior}

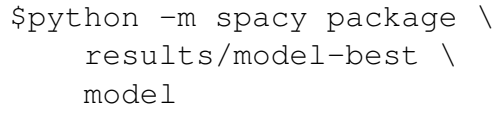

\subsubsection{Etiquetado de un nuevo informe}

Por último, hemos creado un script que utiliza el modelo generado para etiquetar nuevos informes. Como ya hemos comentado con anterioridad, los resultados extraídos por esta funcionalidad deben ser revisados por un experto, que valide aquellas características que considere correctas y establezca las relaciones entre ellas para ser incluidas en la ontología.

\subsubsection{Selección manual}

El último paso consiste en la selección manual de los conceptos sugeridos por el sistema y su inclusión en la ontología. Para ello, basta con incluir los conceptos que finalmente queramos añadir, correctamente colocados en el fichero CSV en base al cuál generamos la ontología, y lanzar el proceso de generación.

Por supuesto, también podemos utilizar cualquier otra herramienta de edición de ontologías para incluir los nuevos casos.

\section{CONCLUSIONES}

En esta investigación hemos propuesto una estructura para una ontología, que es, hasta donde sabemos, pionera en el ámbito del emprendimiento social, hemos presentado un prototipo ilustrativo para el poblado semiautomático $\mathrm{y}$, por último hemos expuesto una función de similitud original definida en el espacio ontológico, núcleo del sistema RBC propuesto.

Con la combinación de Minería de Textos Semántica, Ontologías y sistemas RBC nos hemos adentrado en el campo de la IA que combina enfoques simbólicos y probabilistas, aportando nuestros resultados y experiencias a esta tendencia actual. Además, al aplicarlo al campo del emprendimiento social y la medición de su impacto, nos centramos en un importante ámbito para la consecución de los ODS y en el que apenas se ha trabajado con técnicas de IA.

En primer lugar, hemos podido comprobar que el enfoque de nuestra investigación, basado en la combinación de métodos de IA simbólicos y probabilistas es válido para la resolución de las tareas propuestas. Siendo conscientes de que es necesario profundizar con la realización de más investigaciones, especialmente aumentando el tamaño de la base de casos, consideramos que el apoyo de la minería de textos en el enfoque ontológico y en el sistema RBC es un campo de investigación con muchas posibilidades en base a nuestros resultados.

Modelar el conocimiento sobre el impacto social es factible y útil, el trabajo de investigación llevado a cabo puede ser de gran apoyo para las empresas sociales. La ontología construida ha cumplido con los objetivos del trabajo y se ha adaptado a las tareas para las que ha sido utilizada.

Sobre este punto, cabe destacar que la estructura sugerida está deliberadamente simplificada para facilitar la experimentación. Caben todavía mejoras en su estructura y es posible aumentar el detalle de la misma y completarla con otras ontologías que modelen el conocimiento de alguna de sus ramas.

Respecto al apartado relativo a la minería de textos para el poblado de la ontología, es claramente el apartado más incipiente. Si bien el enfoque NER es de utilidad para algunas características (las fuentes han obtenido buenos resultados), en la mayoría de casos las métricas de evaluación, sobre todo el Recall, no han sido positivas.

Esto puede deberse a varias causas. En primer lugar, el corpus con el que se ha entrenado el sistema es de baja calidad por dos aspectos: por un lado, el etiquetado se ha realizado automáticamente y no es perfecto y, por otro, es demasiado reducido para esta tarea. Es perfectamente conocido que ambas características de nuestros datos de entrenamiento y validación reducen drásticamente la calidad de los resultados.

Por lo tanto, es demasiado pronto para extraer ninguna conclusión definitiva. La repetición del experimento con datos de mayor calidad es imprescindible para confirmar o desmentir cualquier hipótesis. Sería especialmente interesante integrar más casos de entre los recopilados en Social Value UK para obtener resultados más fiables y completos. Hay que tener en cuenta que este apartado no ha sido central en la investigación sino que se trata de un apartado ilustrativo. Además, ya existe abundante investigación en este ámbito en la bibliografía.

Los buenos resultados obtenidos por las funciones de similitud para la recuperación de casos nos hacen pensar que el enfoque RBC es un enfoque válido para la tarea que estamos acometiendo. No obstante, el reducido número de casos utilizados nos obliga a ser prudentes con las conclusiones; para confirmar esta hipótesis son necesarias más investigaciones en este ámbito incluyendo un número mayor de casos y valoraciones por más expertos independientes.

Queda pendiente un estudio de la complejidad del algoritmo y la búsqueda de la optimización de su implementación, si bien estos aspectos no son particularmente relevantes ya que no se trata de una aplicación con requisitos de ejecución en tiempo real.

En resumen, aunque quede trabajo por hacer en el campo que nos ocupa, la investigación llevada a cabo supone una primera incursión en el ámbito de la Inteligencia Artificial aplicada a la medición del impacto social, además de profundizar en el campo de la combinación de diferentes enfoques de IA. En este sentido, los trabajos previos estudiados y los resultados obtenidos en las diferentes partes del sistema nos hacen confiar en la validez de nuestras hipótesis.

Por último, es reseñable la aportación de nuestra investigación no sólo al campo de la IA sino también al del emprendimiento social y la medición de su impacto, con su consecuente interés para los ODS. Además, la versatilidad del sistema propuesto es ideal para continuar la investigación aplicándolo en distintos ámbitos, como en el caso de la propia IA, cuyos sistemas podrían incluir componentes de medición de impacto por diseño integrándose de forma natural en ellos al estar también basados en las técnicas IA mostradas en SIAMES

\section{TRABAJOS FUTUROS}

En primer lugar, si bien la ontología construida y poblada con un número reducido de casos ha cumplido con su cometido experimental, sería interesante enriquecer la ontología, tanto revisando su estructura como poblándola con un número más alto de casos. Los resultados obtenidos nos hacen confiar en la validez de nuestro modelo, por lo que parece lógico que merece la pena invertir esfuerzos en este enriquecimiento para continuar nuestra investigación. Repetir la experimentación realizada y revalidar los resultados con una 
base de casos más amplia sería la primera tarea a realizar.

Respecto a la función de similitud, sus prometedores resultados nos abren la posibilidad de proseguir nuestra investigación evolucionando el sistema recomendador para que sea capaz no sólo de recuperar los casos más similares sino que, dentro de esos casos, sea capaz de recuperar las características más cercanas al nuevo caso que se presente, una línea de trabajo de gran interés.

El poblado semiautomático de la ontología de emprendimiento social plantea retos particulares que es de interés investigar adicionalmente. Para ello es necesario construir un corpus más extenso y de mayor calidad para validar o desmentir la hipótesis sobre el uso de técnicas NER para la extracción. Por otro lado, tampoco se han explorado otras vías para la extracción de información, como los patrones lingüisticos, que podrían resultar de gran interés en esta tarea. No obstante, reiteramos que este apartado no era el central en nuestra investigación y que existen diversos estudios en este ámbito en la bibliografía.

Otro aspecto en el que trabajar sería la orquestación de las diferentes partes de SIAMES para su integración de una manera más eficaz; tanto de cara a simplificar los procesos para la investigación y establecer un marco de trabajo como con vistas a poder utilizar el sistema en entornos reales. El uso de estándares de facto, como puede ser Apache Airflow ${ }^{22}$, para esta tarea sería de interés.

Por último, y como ya avanzábamos en la introducción y las conclusiones, la medición de impacto puede ser aplicada sobre otros ámbitos distintos al emprendimiento social. Especialmente interesante sería abrir una línea de investigación sobre la aplicación del modelo propuesto para la medición de impacto de las propias aplicaciones IA, un campo que consideramos muy prometedor y en el que se ha observado especial interés en diversos ámbitos y organizaciones.

\section{References}

[1] Agnar Aamodt and Enric Plaza, 'Case-based reasoning: Foundational issues, methodological variations, and system approaches', AI Communications, 7, 39-59, (1994).

[2] Malin Arvidson, Fergus Lyon, Stephen McKay, and Domenico Moro, 'The ambitions and challenges of sroi', Third Sector Research Centre. Working paper 49, (12 2010).

[3] Ioanna Boulouta and Christos Pitelis, 'Who needs csr? the impact of corporate social responsibility on national competitiveness', Journal of Business Ethics, 119, (02 2013).

[4] P. Buitelaar, P. Cimiano, and Berenike Litz, 'Proceedings of the 2nd workshop on ontology learning and population: Bridging the gap between text and knowledge', in OntologyLearning@COLING/ACL, (2006).

[5] Ye Cai, Hoje Jo, and Carrie Pan, 'Doing well while doing bad? csr in controversial industry sectors', fournal of Business Ethics, 108, (07 2012).

[6] David Celjuska and Dr. Maria Vargas-Vera, 'Ontosophie: A semiautomatic system for ontology population from text', International Conference on Natural Language Processing (ICON), (01 2004).

[7] Cheng Cheng, Qingtian Zeng, Hua Zhao, Wenyan Guo, and Hua Duan, 'Social impact assessment of storm surge disaster through dynamic neural network model', International fournal of Performability Engineering, 15(10), 2817, (2019).

[8] Albert Hyunbae Cho, Politics, Values and Social Entrepreneurship: A Critical Appraisal, 34-56, Palgrave Macmillan UK, London, 2006.

[9] F. Corcoglioniti, M. Rospocher, and A. P. Aprosio, 'Frame-based ontology population with pikes', IEEE Transactions on Knowledge and Data Engineering, 28(12), 3261-3275, (2016).

[10] Valerio De Stefano, "negotiating the algorithm': Automation, artificial intelligence and labour protection', Comparative labor law journal: a publication of the U.S. National Branch of the International Society for Labor Law and Social Security [and] the Wharton School, and the Law School of the University of Pennsylvania, forthcoming, (06 2018).

22 https://airflow.apache.org
[11] Magali A. Delmas and Vanessa Cuerel Burbano, 'The drivers of greenwashing', California Management Review, 54(1), 64-87, (2011).

[12] Pedro Domingos, The Master Algorithm: How the Quest for the Ultimate Learning Machine Will Remake Our World, Basic Books, Inc., USA, 2018.

[13] D. Dou, H. Wang, and H. Liu, Semantic data mining: A survey of ontology-based approaches, Proceedings of the 2015 IEEE 9th International Conference on Semantic Computing (IEEE ICSC 2015), 2015.

[14] Valmi Dufour-Lussier, Florence Le Ber, Jean Lieber, and Emmanuel Nauer, 'Automatic case acquisition from texts for process-oriented case-based reasoning', Information Systems, 40, 153 - 167, (2014).

[15] A. Ebrahim, J. Battilana, and J. Mair, The governance of social enterprises: mission drift and accountability challenges in hybrid organizations, volume 34, Research in Organizational Behavior, 2014.

[16] A.M. Eikenberry and J.D. Kluver, The marketization of the nonprofit sector: civil society at risk?, volume 64 (2), Public Administration Review, 2004.

[17] Shaker El-Sappagh and Mohammed Elmogy, 'Case based reasoning: Case representation methodologies', International fournal of Advanced Computer Science and Applications, 6, 192-208, (11 2015).

[18] M. Fernández-López, Asunción Gómez-Pérez, and N. Juristo, 'Methontology: From ontological art towards ontological engineering', in $A A A I$ 1997, (1997).

[19] Blaz Fortuna, Marko Grobelnik, and Dunja Mladenic, 'Ontogen: Semiautomatic ontology editor', in Human Interface and the Management of Information. Interacting in Information Environments, eds., Michael J. Smith and Gavriel Salvendy, pp. 309-318, Berlin, Heidelberg, (2007). Springer Berlin Heidelberg.

[20] Daniel Greene, Anna Lauren Hoffmann, and Luke Stark, 'Better, nicer, clearer, fairer: A critical assessment of the movement for ethical artificial intelligence and machine learning', in HICSS, (2019).

[21] Wu He, 'Improving user experience with case-based reasoning systems using text mining and web 2.0', Expert Systems with Applications, 40(2), $500-507,(2013)$.

[22] Stephen Healy, Kelly Dombroski, David Conradson, Gradon Diprose, and Joanne McNeilland Alison Watkins, 'More than monitoring: Developing impact measures for transformative social enterprise', $U N$ SEE, (2019).

[23] Robert Hoehndorf and Núria Queralt-Rosinach, 'Data science and symbolic ai: Synergies, challenges and opportunities', Data Science, 112, (06 2017).

[24] Dirk Hovy and Shannon L. Spruit, 'The social impact of natural language processing', in Proceedings of the 54th Annual Meeting of the Association for Computational Linguistics (Volume 2: Short Papers), pp. 591-598, Berlin, Germany, (August 2016). Association for Computational Linguistics.

[25] Hoje Jo and Haejung $\mathrm{Na}$, 'Does csr reduce firm risk? evidence from controversial industry sectors', fournal of Business Ethics, 110, (11 2012).

[26] Agnieszka Konys, 'An ontology-based knowledge modelling for a sustainability assessment domain', Sustainability, 10, 300, (01 2018).

[27] A. Kroeger and C. Weber, Developing a conceptual framework for comparing social value creation, volume 39, Academy of Management Review, 2014.

[28] Yehong Li, Raúl García-Castro, Nandana Mihindukulasooriya, James O’Donnell, and Sergio Vega-Sánchez, 'Enhancing energy management at district and building levels via an em-kpi ontology', Automation in Construction, 99, 152 - 167, (2019).

[29] D. Littlewood and D. Holt, 'How social enterprises can contribute to the sustainable development goals (sdgs) - a conceptual framework', Entrepreneurship and the Sustainable Development Goals (Contemporary Issues in Entrepreneurship Research), 8, 33-46, (2018).

[30] Mohamed Lubani, Shahrul Azman Mohd Noah, and Rohana Mahmud, 'Ontology population: Approaches and design aspects', Journal of Information Science, 45(4), 502-515, (2019).

[31] Alessandro Mantelero, 'Ai and big data: A blueprint for a human rights, social and ethical impact assessment', Computer Law \& Security Review, 34(4), 754 - 772, (2018).

[32] Yoshihiro Maruyama, 'The categorical integration of symbolic and statistical ai: Quantum nlp and applications to cognitive and machine bias problems', in Intelligent Systems Design and Applications, eds., Ajith Abraham, Patrick Siarry, Kun Ma, and Arturas Kaklauskas, pp. 466476, Cham, (2021). Springer International Publishing.

[33] Diana Maynard, Adam Funk, and Wim Peters, 'Sprat: a tool for automatic semantic pattern-based ontology population', in IN: INTERNA- 
TIONAL CONFERENCE FOR DIGITAL LIBRARIES AND THE SEMANTIC WEB, (2009).

[34] Ross Millar and Kelly Hall, 'Social return on investment (sroi) and performance measurement', Public Management Review, 15(6), 923-941, (2013).

[35] Jerry Muller, The Tyranny of Metrics, Princeton University Press, 02 2018.

[36] A. Nicholls, 'We do good things, don't we?': Blended value accounting in social entrepreneurship, volume 34, Accounting, Organizations and Society, 2009.

[37] Georgios Petasis, Vangelis Karkaletsis, Georgios Paliouras, Anastasia Krithara, and Elias Zavitsanos, Ontology Population and Enrichment: State of the Art, 134-166, Springer Berlin Heidelberg, Berlin, Heidelberg, 2011.

[38] Amir Rahdari, Sahar Sepasi, and Mohammad Moradi, 'Achieving sustainability through schumpeterian social entrepreneurship: The role of social enterprises', fournal of Cleaner Production, 137, 347 - 360, (2016).

[39] Juana Ruiz-Martínez, Jose Antonio Miñarro Giménez, Dagoberto Nieves, Francisco Garcia-Sanchez, and Rafael Valencia-García, 'Ontology population: An application for the e-tourism domain', International Journal of Innovative Computing, Information and Control, 7, (11 2011).

[40] Juana Ruiz-Martínez, Rafael Valencia-García, Rodrigo Martínez-Béjar, and Achim Hoffmann, 'Bioontoverb: A top level ontology based framework to populate biomedical ontologies from texts', Knowledge-Based Systems, 36, 68-80, (12 2012).

[41] Stefan Schaltegger, Markus Beckmann, and Kai Hockerts, 'Collaborative entrepreneurship for sustainability. creating solutions in light of the un sustainable development goals', International fournal of Entrepreneurial Venturing, 10, 131, (01 2018).

[42] Chung-Hua Shen and Yuan Chang, 'Ambition versus conscience, does corporate social responsibility pay off? the application of matching methods', Journal of Business Ethics, 88, 133-153, (04 2008).

[43] Liyin Shen, Hang Yan, Hongqin Fan, Ya Wu, and Yu Zhang, 'An integrated system of text mining technique and case-based reasoning (tmcbr) for supporting green building design', Building and Environment, 124, 388 - 401, (2017)

[44] Joanna Stempczyńska and Edward Kącki, Artificial Inteligence Methods in the Problems of Drug Side-Effects, 123-127, Springer Berlin Heidelberg, Berlin, Heidelberg, 2009.

[45] Xue Xiao, Martin Skitmore, and Xin Hu, 'Case-based reasoning and text mining for green building decision making', Energy Procedia, 111, 417 - 425, (2017). 8th International Conference on Sustainability in Energy and Buildings, SEB-16, 11-13 September 2016, Turin, Italy.

[46] Jieyu Zhao, Tianlu Wang, Mark Yatskar, Vicente Ordonez, and Kai-Wei Chang. Men also like shopping: Reducing gender bias amplification using corpus-level constraints, 2017. 


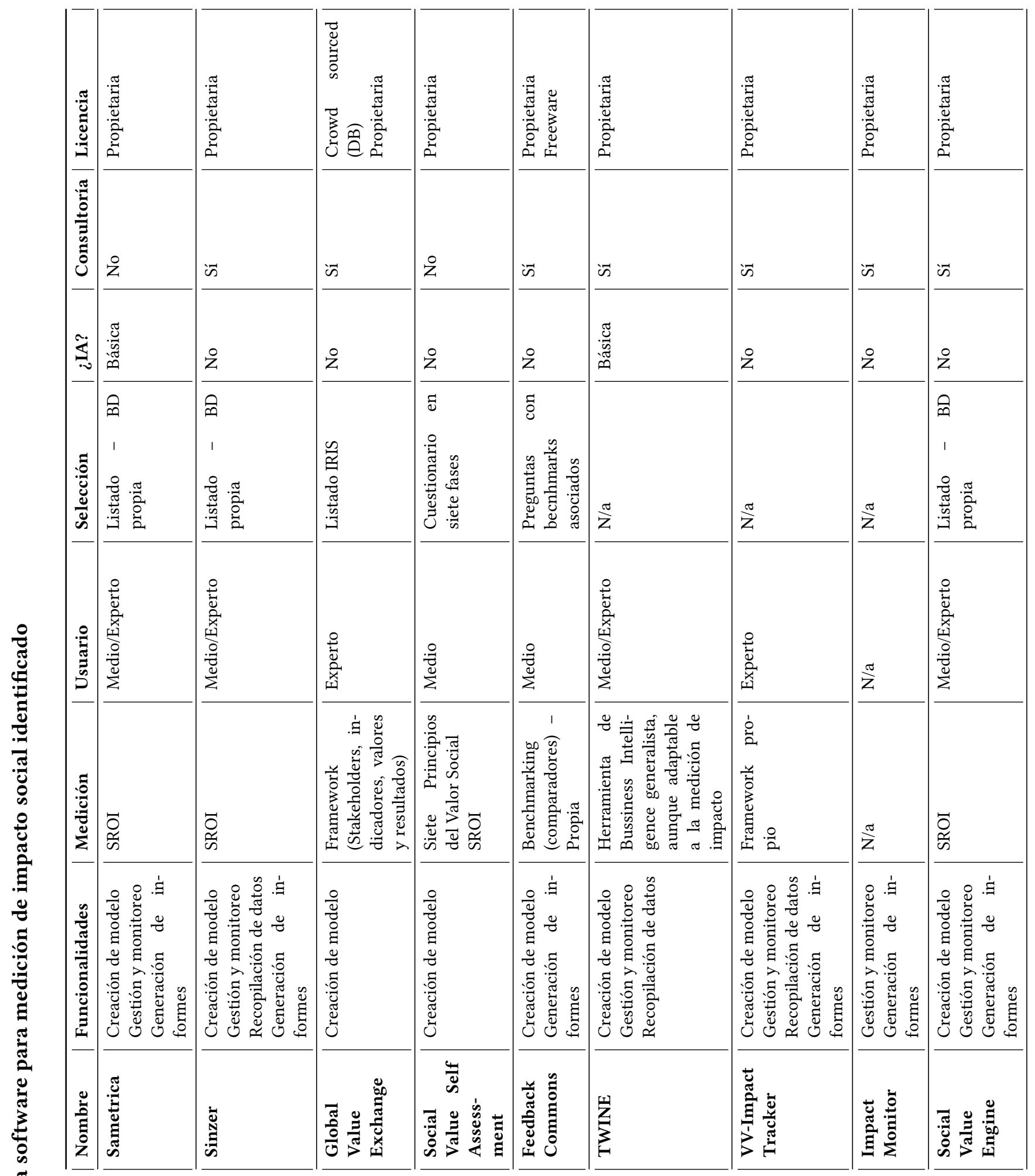




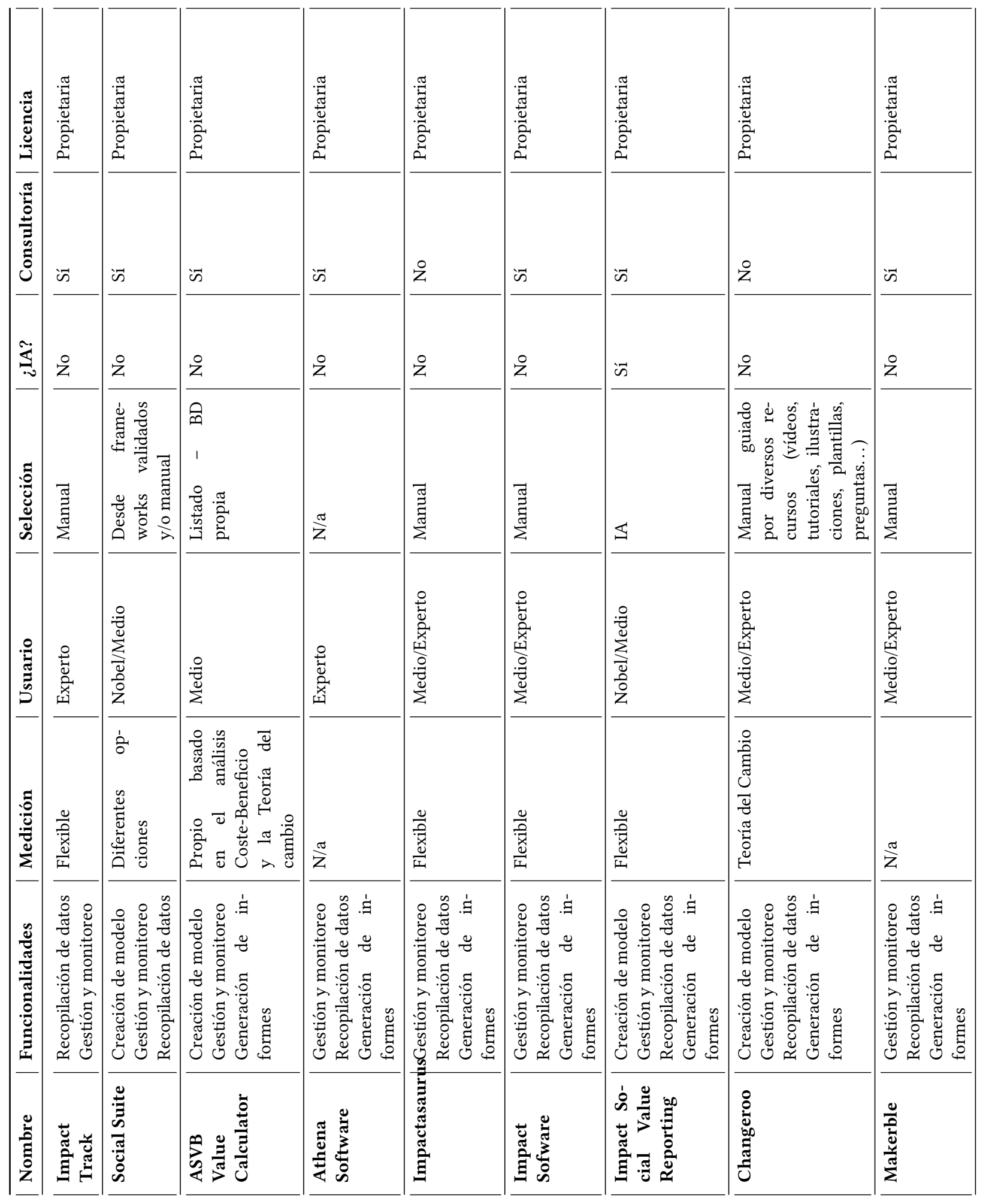




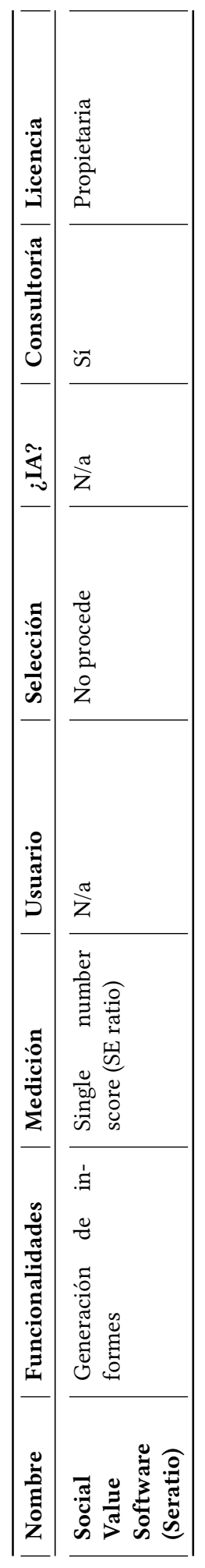




\section{B. Resultados completos función de similitud}

\begin{tabular}{|c|c|c|}
\hline case 1 & case2 & score \\
\hline 001 & 012 & 0.6211733361085257 \\
\hline 001 & 013 & 0.5994900027910868 \\
\hline 001 & 006 & 0.5872899949550628 \\
\hline 001 & 014 & 0.5714466700951258 \\
\hline 001 & 002 & 0.5635499993960064 \\
\hline 001 & 003 & 0.5279066661993662 \\
\hline 001 & 011 & 0.4975100000699361 \\
\hline 001 & 009 & 0.48828333020210263 \\
\hline 001 & 008 & 0.47291000187397003 \\
\hline 001 & 004 & 0.44958666761716204 \\
\hline 001 & 007 & 0.4099633350968361 \\
\hline 002 & 006 & 0.7469013722796066 \\
\hline 002 & 001 & 0.6346722525416636 \\
\hline 002 & 013 & 0.6199546044948053 \\
\hline 002 & 003 & 0.5966485316262525 \\
\hline 002 & 008 & 0.5811100884103307 \\
\hline 002 & 012 & 0.5535229385483499 \\
\hline 002 & 014 & 0.5422926378600738 \\
\hline 002 & 011 & 0.516931573812868 \\
\hline 002 & 009 & 0.510931473386054 \\
\hline 002 & 004 & 0.4784781347595009 \\
\hline 002 & 007 & 0.44308471029525043 \\
\hline 003 & 009 & 0.738947874608666 \\
\hline 003 & 011 & 0.7289402052609608 \\
\hline 003 & 007 & 0.7036315189166502 \\
\hline 003 & 014 & 0.6393660556186329 \\
\hline 003 & 002 & 0.6021721215561183 \\
\hline 003 & 004 & 0.5895028244967413 \\
\hline 003 & 013 & 0.589299394205363 \\
\hline 003 & 006 & 0.5864800021503911 \\
\hline 003 & 001 & 0.5642828313991276 \\
\hline 003 & 012 & 0.5550202038553026 \\
\hline 003 & 008 & 0.48692727191279633 \\
\hline 004 & 014 & 0.5554441950574156 \\
\hline 004 & 003 & 0.46108663390851956 \\
\hline 004 & 009 & 0.45466950670220796 \\
\hline 004 & 013 & 0.4449110713444258 \\
\hline 004 & 011 & 0.4406824539428765 \\
\hline 004 & 008 & 0.42244043189381797 \\
\hline 004 & 002 & 0.41265175113751673 \\
\hline 004 & 001 & 0.412242939647477 \\
\hline 004 & 006 & 0.38742384856057943 \\
\hline 004 & 012 & 0.37495647419365424 \\
\hline 004 & 007 & 0.3747375089871256 \\
\hline 005 & 010 & 0.5892316223549368 \\
\hline 006 & 002 & 0.7173924615176824 \\
\hline 006 & 001 & 0.6297679447019712 \\
\hline 006 & 013 & 0.606790059317763 \\
\hline 006 & 014 & 0.5952629804229125 \\
\hline 006 & 003 & 0.5899067301398668 \\
\hline 006 & 012 & 0.5749181037625442 \\
\hline 006 & 011 & 0.5598546476222767 \\
\hline 006 & 009 & 0.5532596121804837 \\
\hline 006 & 008 & 0.5296007947375377 \\
\hline 006 & 004 & 0.5049995166273453 \\
\hline 006 & 007 & 0.494333492186016 \\
\hline 007 & 003 & 0.7407244424025218 \\
\hline 007 & 009 & 0.6944244470861224 \\
\hline 007 & 011 & 0.6934266693062252 \\
\hline 007 & 014 & 0.5614822235372331 \\
\hline 007 & 006 & 0.5480644490983752 \\
\hline 007 & 013 & 0.541935556464725 \\
\hline 007 & 002 & 0.5343622280491723 \\
\hline 007 & 012 & 0.5131266620424059 \\
\hline
\end{tabular}

\begin{tabular}{|c|c|c|}
\hline & 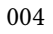 & \\
\hline & 001 & \\
\hline & 008 & 70 \\
\hline & 002 & 0.5129688841601213 \\
\hline & 14 & .4770244417091211 \\
\hline & 13 & .4718322238160504 \\
\hline & 06 & .4695877736641301 \\
\hline & 001 & 500678 \\
\hline & 03 & 0.440366662459241 \\
\hline 8 & 12 & 5557166 \\
\hline & 004 & 0.40800 \\
\hline & 11 & 873 \\
\hline 8 & 009 & 2880357 \\
\hline & 007 & 756769 \\
\hline & 011 & 95076 \\
\hline 9 & 03 & 94944 \\
\hline 99 & 007 & 937727 \\
\hline 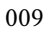 & 014 & 0.5648 \\
\hline & 013 & 4462 \\
\hline 9 & 004 & 7804 \\
\hline & 006 & \\
\hline & 02 & 839 \\
\hline & 01 & \\
\hline & 12 & \\
\hline J9 & 008 & \\
\hline & 05 & 0.6292 \\
\hline & 009 & \\
\hline & 003 & 1205 \\
\hline 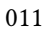 & 007 & 315 \\
\hline & 014 & 3877 \\
\hline & 006 & \\
\hline & 001 & 1288 \\
\hline 01 & 013 & \\
\hline 1 & 02 & \\
\hline & 04 & 2981 \\
\hline & 012 & \\
\hline & 08 & \\
\hline & 01 & 27 \\
\hline & 002 & 36813 \\
\hline & 013 & \\
\hline 12 & 03 & \\
\hline & 006 & 335 \\
\hline 01 & 14 & 912182 \\
\hline 012 & 009 & 08027 \\
\hline 2 & 11 & 359 \\
\hline & & 5869 \\
\hline 012 & 008 & 3007246 \\
\hline 012 & 07 & 5583 \\
\hline 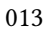 & 01 & 251 \\
\hline & & 525018 \\
\hline & & \\
\hline 013 & 03 & 86906 \\
\hline 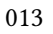 & 02 & 04746 \\
\hline & & 936058 \\
\hline 01 & & \\
\hline 013 & 9 & 26287 \\
\hline & 008 & 0.4937 \\
\hline & & \\
\hline 01 & 7 & 1044 \\
\hline 014 & 004 & 4957 \\
\hline 01 & & 6672404 \\
\hline & & \\
\hline 014 & 001 & 0.5773237934512134 \\
\hline 014 & & 0.5743478366796778 \\
\hline 01 & 列 & 1260740 \\
\hline 01 & 00 & 750497 \\
\hline 014 & 002 & 0.5170192346462675 \\
\hline 01 & 012 & 0.5104298560156306 \\
\hline 014 & $00^{\prime}$ & 0.4941674046672804 \\
\hline
\end{tabular}

